\title{
Dependence of the superconducting critical temperature on the number of layers in homologous series of high- $T_{c}$ cuprates
}

\author{
T. A. Zaleski and T. K. Kopeć \\ Institute of Low Temperature and Structure Research, \\ Polish Academy of Sciences \\ P.O. Box 1410, 50-950 Wroctaw, Poland
}

\begin{abstract}
We study a model of $n$-layer high-temperature cuprates of homologous series like $\mathrm{HgBa}_{2} \mathrm{Ca}_{n-1} \mathrm{Cu}_{n} \mathrm{O}_{2+2 n+\delta}$ to explain the dependence of the critical temperature $T_{c}(n)$ on the number $n$ of $\mathrm{Cu}-\mathrm{O}$ planes in the elementary cell. Focusing on the description of the high-temperature superconducting system in terms of the collective phase variables, we have considered a semi-microscopic anisotropic three-dimensional vector XY model of stacked copper-oxide layers with adjustable parameters representing microscopic in-plane and out-of-plane phase stiffnesses. The model captures the layered composition along $c$-axis of homologous series and goes beyond the phenomenological Lawrence-Doniach model for layered superconductors. Implementing the spherical closure relation for vector variables we have solved the phase XY model exactly with the help of transfer matrix method and calculated $T_{c}(n)$ for arbitrary block size $n$, elucidating the role of the $c$-axis anisotropy and its influence on the critical temperature. Furthermore, we accommodate inhomogeneous charge distribution among planes characterized by the charge imbalance coefficient $R$ being the function of number of layers $n$. By making a physically justified assumption regarding the doping dependence of the microscopic phase stiffnesses, we have calculated the values of parameter $R$ as a function of block size $n$ in good agreement with the nuclear magnetic resonance data of carrier distribution in multilayered high- $T_{c}$ cuprates.
\end{abstract}

\section{INTRODUCTION}

Characteristic common structure of high-temperature superconducting cuprates has been puzzling researchers since the discovery of the phenomenon by Bednorz and Müller $\frac{1}{-}$ It is now accepted that all relevant carriers of both spin and electricity derive from the hybridized antibonding orbitals in the $\mathrm{Cu}-\mathrm{O}$ planes ${ }_{2}^{2}$ Obviously, the interlayer structure cannot be ignored ${ }^{3}$ In the normal state, due to huge anisotropy, coherent transport in the direction perpendicular to $\mathrm{Cu}-\mathrm{O}$ planes is blocked. However, it is observed that $T_{c}$ is strongly dependent on the interlayer structure, which means that threedimensional (3D) coupling of planes must play an important role in the onset of superconductivity ${ }^{\underline{4}}$ It suggested to apply the Lawrence and Doniach model (LD) to cuprates, which originally was proposed for layered superconductors and is based on the Ginzburg-Landau description with neighboring layers coupled by the Josephson tunneling, ${ }^{\frac{5}{5}}$ This approach was pursued to study Josephson plasma resonance,$\frac{6}{}$ anisotropy of critical fields $H_{c 1}$ and $H_{c 2} \stackrel{7.8}{~ v o r t e x ~ m a t t e r ~ b e h a v i o r ~}{ }^{9}$ However, the biggest disadvantage of the LD model is that it is based on mean-field approximation, which neglects the role of phase fluctuations, the latter playing a profound role in cuprates $\underline{\underline{10}}$

There is a significant evidence that in cuprates the $\mathrm{Cu}-\mathrm{O}$ planes are Josephson coupled and the anisotropy of $c$-axis to $a b$-planes penetration depth can vary from 5 to even 250 in various high- $T_{c}$ compounds. This gave rise to interplane theories e.g. interlayer tunneling model (ILT) by Anderson, 11 which assumed that carriers were incoherent between layers in the normal phase, however coherent transport of Cooper pairs in a form of
Josephson tunneling in superconducting state was allowed. Unfortunately, the original version of the ILT theory by Anderson is no longer considered as a viable model of high- $T_{c}$ materials, since it provides no more than $1 \%$ of the condensation energy in certain cuprates $\underline{12,13}$ However, the Josephson coupling between $\mathrm{Cu}-\mathrm{O}$ planes is still believed to play an important role in cuprate oxides $\underline{14}$ In the process of synthesis of high- $T_{c}$ materials it is possible to control (to some extent) the distances between copper-oxide planes or the content of the regions that separate them. It allows to study, how such changes influence observed properties of these compounds like critical temperature or electrical resistivity and elucidate the role of the inter-layer coupling. ${ }^{15.16}$ In mono- or bi-layered high- $T_{c}$ materials (like LSCO or YBCO compounds), all copper-oxide planes are equivalent, which means that charge carriers are distributed homogeneously among copper-oxide planes. However, multi-layered high- $T_{c}$ materials are characterized by the existence of crystalographically inequivalent outer (OP) and inner (IP) $\mathrm{Cu}-\mathrm{O}$ planes, which give rise to inhomogeneous carrier distribution among them. This, in turn, may have a profound influence on the critical temperature, which is know to be strongly doping dependent. For example, high-temperature superconducting homologous series, like $\mathrm{HgBa}_{2} \mathrm{Ca}_{n-1} \mathrm{Cu}_{n} \mathrm{O}_{2+2 n+\delta}$, are materials sharing the same charge reservoir, but differing by the number $n$ of $\mathrm{Cu}-\mathrm{O}$ planes in the unit cell $\frac{17.18}{18}$ They also have the same characteristic dependence of the critical temperature as a function of $n$ with a maximum for $n=3$ or $n=4$. Although, these groups of cuprates have been extensively studied, the origin of this extremum is still not fully understood 19.20 .21

Recently, Chakravarty et al. have argued that the ex- 
istence of the competing order parameter that is nucleated by charge imbalance is ultimately responsible for the critical temperature downturn with $n i^{22}$ They present a mean-field theory for a single block of $n$ superconducting layers, however neglecting the inter-block coupling. Moreover, they use phenomenological Ginzburg-Landau theory at zero-temperature to anticipate the values of the critical temperature $T_{c}(n)$, based on the magnitude of the $T=0$ superconducting order parameter. Charge imbalance is introduced as a competing order parameter, e.g. $d$-density wave ${ }^{23}$ It depresses the critical temperature for $n>2$, thus experimentally observed shape of $T_{c}(n)$ is obtained. However, as the authors note, the phase fluctuations, which are not taken into account, might depress $T_{c}$ for $n=1$ even more than for $n=3$. Although, it should correct the results for $1<n<3$, it is not obvious, whether the maximum of $T_{c}(n)$ can be observed at all.

It is well known that binding of electrons into pairs is essential in forming the superconducting state, however, its remarkable properties - zero resistance and Meissner effect - require the phase coherence among the pairs as well. In conventional BCS superconductors the phase order is unimportant for determining of the value of the transition temperature $T_{c}$. However, in superconductors with low carrier density such as high- $T_{c}$ oxide superconductors, phase fluctuations may have a profound influence on low temperature properties $\stackrel{10}{\underline{10}}$ In particular, for cuprate superconductors, the conventional ordering of binding and phase stiffness energies appears to be reversed. Thus, the issue how pairing and phase correlations develop is a central problem of high- $T_{c}$ superconductivity. The measurements of the frequency dependent conductivity, in the frequency range $100-600 \mathrm{GHz}$, shows that phase correlations indeed persist above $T_{c}$, where the phase dynamics is governed by the bare microscopic phase stiffnesses $\underline{24}^{24}$

In the present paper we propose a semi-microscopic model of $n$-layer cuprates, which is founded on microscopic phase stiffnesses that set the characteristic energy scales: in-plane $J_{\|}$, inter-plane (in-block) $J_{\perp}$ and inter-block $J_{\perp}^{\prime}$ couplings. We employ an approach that goes beyond the mean field level and is able to capture both the effects of phase fluctuations and huge $c$ axis anisotropy on the superconducting phase transition. We show that the depression of the critical temperature for $n>3$ can be obtained naturally without invoking any competing (hidden) order. Since superconducting carriers are located in $\mathrm{Cu}-\mathrm{O}$ planes, the in-plane phase stiffness must naturally be doping $\delta$ dependent. As this dependence can be simply deduced from the established $T_{c}-\delta$ phase diagram for cuprates, introduction of charge imbalance via the doping dependence of microscopic inplane phase stiffness allows us for explanation of $T_{c}(n)$ as a function of block layers $n$ in perfect agreement with experimental findings $\frac{17}{17}$

The outline of the reminder of the paper is as follows. In Section II we analyze a single-layer system. We con- struct an anisotropic three-dimensional XY model, which will be solved in the spherical approximation to explicate the dependence of critical temperature on the anisotropy $\eta=J_{\perp} / J_{\|}$along $c$-axis. Subsequently, in Section III we elaborate on a model of stacked $n$-block layers relevant for high-temperature superconducting homologous series. We map the system onto a spherical XY model, which is solved exactly for arbitrary $n$ with the help of the transfer matrix method. In Section IV we determine the critical temperature $T_{c}(n)$ as a function of number of layers $n$. In Section $\mathrm{V}$ we incorporate the inhomogeneous charge distribution in homologous series by introducing doping dependent in-plane phase stiffnesses $J_{\|}(\delta)$ for the separate $n$ layers in the block structure. We found that the maximum of the phase coherent superconducting transition will be a bell-shaped curve as a function of $n$ with a maximum for $n=3$ for appropriate values of outer $\left(\delta_{o p}\right)$ and inner $\left(\delta_{i p}\right)$ planes dopings within a block. Finally, in Section VI we summarize the conclusions to be drawn from our work. Some supplementary material is relegated to the Appendix.

\section{SINGLE LAYER CUPRATES}

In underdoped high-temperature superconductors, two temperature scales of short-length pairing correlations and long-range superconducting order seem to be well separated 10 . We consider the situation, in which local superconducting pair correlations are established and the relevant degrees of freedom are represented by phase factors $0 \leq \varphi_{\ell}\left(\mathbf{r}_{i}\right)<2 \pi$, where $\mathbf{r}_{i}$ numbers lattice sites within $\ell$-th $a b$ plane. The system becomes superconducting once U(1) symmetry group governing the $\varphi_{\ell}\left(\mathbf{r}_{i}\right)$ factors is spontaneously broken and the non-zero value of $\left\langle e^{i \varphi_{\ell}\left(\mathbf{r}_{i}\right)}\right\rangle$ appears signaling the long-range phase order. The dependence of critical temperature on the spatial $c$ axis anisotropy is of paramount importance for cuprate systems, in which two-dimensional physics predominate. First, we consider a single-layer system described by the following Hamiltonian:

$$
\begin{aligned}
H[\varphi]= & -J_{\|} \sum_{\ell} \sum_{i<j} \cos \left[\varphi_{\ell}\left(\mathbf{r}_{i}\right)-\varphi_{\ell}\left(\mathbf{r}_{j}\right)\right] \\
& -J_{\perp} \sum_{\ell} \sum_{i} \cos \left[\varphi_{\ell}\left(\mathbf{r}_{i}\right)-\varphi_{\ell+1}\left(\mathbf{r}_{i}\right)\right],
\end{aligned}
$$

where $J_{\|}>0$ and $J_{\perp}>0$ are in-plane and inter-plane microscopic phase stiffnesses, respectively. The factors $\varphi_{\ell}\left(\mathbf{r}_{i}\right)$ are placed in sites of a three-dimensional cubic lattice, where $i=1, \ldots, N_{\|}$with $N_{\|}$being the number of sites in a plane, $\ell=1, \ldots, N_{\perp}$, where $N_{\perp}$ denotes the number of layers and $N=N_{\|} N_{\perp}$ is the total number of sites. The partition function of the system reads:

$$
Z=\int_{0}^{2 \pi} \prod_{\ell, i} d \varphi_{\ell}\left(\mathbf{r}_{i}\right) e^{-\beta H[\varphi]},
$$




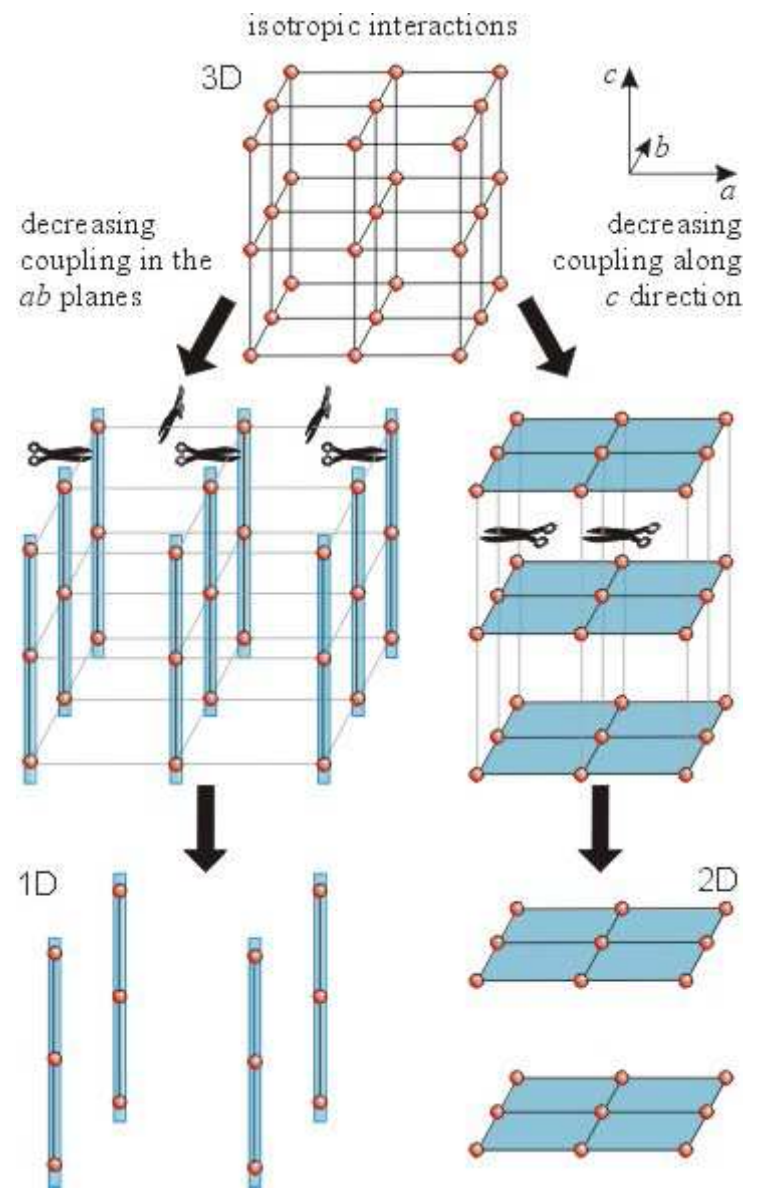

Figure 1: (Color online) Pictorial representation of the various crossovers due to increased spatial anisotropy between in-plane and inter-plane couplings: $3 \mathrm{D} \rightarrow 2 \mathrm{D}$ (isotropic interactions to uncoupled planes) and $3 \mathrm{D} \rightarrow 1 \mathrm{D}$ (isotropic interactions to uncoupled chains).

where $\beta=1 / k_{B} T$ with $T$ being the temperature. Introducing two-dimensional vectors $\mathbf{S}_{\ell}\left(\mathbf{r}_{i}\right)=$ $\left[S_{x \ell}\left(\mathbf{r}_{i}\right), S_{y \ell}\left(\mathbf{r}_{i}\right)\right]$ of the unit length $\mathbf{S}_{\ell}^{2}\left(\mathbf{r}_{i}\right)=S_{x \ell}^{2}\left(\mathbf{r}_{i}\right)+$ $S_{y \ell}^{2}\left(\mathbf{r}_{i}\right)=1$ defined by

$$
\mathbf{S}_{\ell}\left(\mathbf{r}_{i}\right)=\left[\cos \varphi_{\ell}\left(\mathbf{r}_{i}\right), \sin \varphi_{\ell}\left(\mathbf{r}_{i}\right)\right],
$$

the Hamiltonian can be written in the XY-model form:

$$
\begin{aligned}
H[\mathbf{S}]= & -J_{\|} \sum_{\ell} \sum_{i<j} \mathbf{S}_{\ell}\left(\mathbf{r}_{i}\right) \cdot \mathbf{S}_{\ell}\left(\mathbf{r}_{j}\right) \\
& -J_{\perp} \sum_{\ell} \sum_{i} \mathbf{S}_{\ell}\left(\mathbf{r}_{i}\right) \cdot \mathbf{S}_{\ell+1}\left(\mathbf{r}_{i}\right) .
\end{aligned}
$$

In terms of the vector variables the partition function in Eq. (21) becomes:

$$
Z=\int_{-\infty}^{+\infty} \prod_{i, \ell}\left\{d^{2} \mathbf{S}_{\ell}\left(\mathbf{r}_{i}\right) \delta\left[\mathbf{S}_{\ell}^{2}\left(\mathbf{r}_{i}\right)-1\right]\right\} e^{-\beta H[\mathbf{S}]},
$$

where $d^{2} \mathbf{S}_{\ell}\left(\mathbf{r}_{i}\right) \equiv d S_{x \ell}\left(\mathbf{r}_{i}\right) d S_{y \ell}\left(\mathbf{r}_{i}\right)$ and the Dirac- $\delta$ function $\delta\left[\mathbf{S}_{\ell}^{2}\left(\mathbf{r}_{i}\right)-1\right]$ assures that the integration over
$\mathbf{S}_{\ell}\left(\mathbf{r}_{i}\right)$ variables runs only over the values, which satisfy the unit length condition $\mathbf{S}_{\ell}^{2}\left(\mathbf{r}_{i}\right)=1$. Unfortunately, the partition function in Eq. (5) cannot be evaluated exactly. However, replacing of the rigid length constraint in Eq. (5) by a weaker spherical closure relation ${ }^{25.26}$

$$
\delta\left[\mathbf{S}_{\ell}^{2}\left(\mathbf{r}_{i}\right)-1\right] \rightarrow \delta\left[\frac{1}{N} \sum_{i, \ell} \mathbf{S}_{\ell}^{2}\left(\mathbf{r}_{i}\right)-1\right] .
$$

renders the model in Eq. (44) exactly solvable. The relation in Eq. (6) means that the unit length of the $\mathbf{S}_{\ell}\left(\mathbf{r}_{i}\right)$ vectors is maintained on average. The spherical constraint can be conveniently implemented with the help of Dirac- $\delta$ function and the partition function in Eq. (5) becomes:

$$
Z=\int_{-\infty}^{+\infty} \prod_{i, \ell} d^{2} \mathbf{S}_{\ell}\left(\mathbf{r}_{i}\right) \delta\left[\sum_{i, \ell} \mathbf{S}_{\ell}^{2}\left(\mathbf{r}_{i}\right)-N\right] e^{-\beta H[\mathbf{S}]} .
$$

Furthermore, the $\delta$-function can be conveniently represented in a spectral form by the appropriate integral $\delta(x)=\int_{-\infty}^{\infty} d \zeta / 2 \pi i \exp (-\zeta x)$, which introduces the Lagrange multiplier $\zeta$. Consequently, Eq. (7) can be written as:

$$
Z=\int_{-\infty}^{+\infty} \frac{d \zeta}{2 \pi i} \exp [-N \beta f(\zeta)]
$$

where the free energy per site is given by:

$$
\begin{aligned}
f(\zeta) & =-\frac{\zeta}{\beta}-\frac{1}{N \beta} \ln \int_{-\infty}^{+\infty} \prod_{i, \ell} d^{2} \mathbf{S}_{\ell}\left(\mathbf{r}_{i}\right) \\
& \times \exp \left\{-\zeta \sum_{i, \ell} \mathbf{S}_{\ell}^{2}\left(\mathbf{r}_{i}\right)-\beta H[\mathbf{S}]\right\} .
\end{aligned}
$$

In the thermodynamic limit $N \rightarrow \infty$ the dominant contribution to the integral in Eq. (8) comes from the saddle point $\zeta=\zeta_{0}$ of $f(\zeta)$ with value of $\zeta_{0}$ determined from the condition:

$$
\left.\frac{\partial f(\zeta)}{\partial \zeta}\right|_{\zeta=\zeta_{0}}=0
$$

To evaluate the free energy of the system $f=f\left(\zeta_{0}\right)$ we diagonalize the Hamiltonian from Eq. (44) by introducing Fourier transform of $\mathbf{S}_{\ell}\left(\mathbf{r}_{i}\right)$ variables:

$$
\mathbf{S}_{\ell}\left(\mathbf{r}_{i}\right)=\frac{1}{N_{\|} N_{\perp}} \sum_{\mathbf{k}, q_{z}} \mathbf{S}_{\mathbf{k}, q_{z}} e^{-i\left(\mathbf{k r}_{i}+q_{z} c \ell\right)}
$$

where $\mathbf{k}=\left(k_{x}, k_{y}\right)$ with $-\frac{\pi}{a}<k_{x}, k_{y}<\frac{\pi}{a}$ are wave vectors within $a b$-planes, while $-\frac{\pi}{c}<q_{z}<\frac{\pi}{c}$ is wave vector along $c$-axis. Here, $a$ and $c$ are lattice constant within $a b$ plane and $c$-axis, respectively. The Hamiltonian in Eq. (44) then transforms to:

$$
H=-\frac{1}{2 N} \sum_{\mathbf{k}, q_{z}} J\left(\mathbf{k}, q_{z}\right) \mathbf{S}_{\mathbf{k}, q_{z}} \cdot \mathbf{S}_{-\mathbf{k},-q_{z}}
$$


where for the Fourier-transformed interactions $J_{\|}$and $J_{\perp}$ on the 3D anisotropic cubic lattice with nearest-neighbor coupling we readily find:

$$
J\left(\mathbf{k}, q_{z}\right)=2 J_{\|}\left[\cos \left(a k_{x}\right)+\cos \left(a k_{y}\right)\right]+2 J_{\perp} \cos \left(c q_{z}\right) .
$$

As a result, the integral in Eq. (9) becomes Gaussian and the free energy can be calculated:

$$
\begin{aligned}
f= & -\frac{\zeta_{0}}{\beta}-\frac{1}{\beta N} \ln \int_{-\infty}^{+\infty} \prod_{\mathbf{k}, q_{z}} d^{2} \mathbf{S}_{\mathbf{k}, q_{z}} \\
& \times \exp \left\{-\frac{1}{N} \sum_{\mathbf{k}, q_{z}}\left[\zeta_{0}-\frac{1}{2} J\left(\mathbf{k}, q_{z}\right)\right] \mathbf{S}_{\mathbf{k}, q_{z}}^{*} \mathbf{S}_{\mathbf{k}, q_{z}}\right\} \\
& d^{2} \mathbf{S}_{\mathbf{k}, q_{z}} \equiv \frac{d \operatorname{Re} \mathbf{S}_{\mathbf{k}, q_{z}}^{*} d \operatorname{Im} \mathbf{S}_{\mathbf{k}, q_{z}}}{\pi} .
\end{aligned}
$$

The emergence of the critical point in the spherical model is signaled by the condition 25

$$
G^{-1}\left(\mathbf{k}=0, q_{z}=0\right) \equiv \zeta_{0}-\frac{1}{2} \beta J_{0}=0,
$$

which fixes the saddle-point value of the Lagrange multiplier $\zeta$ within the ordered phase:

$$
\zeta_{0}=\frac{1}{2} \beta J_{0},
$$

where $J_{0}=J\left(\mathbf{k}=0, q_{z}=0\right)$. Here, $G\left(\mathbf{k}, q_{z}\right)$ is the order parameter susceptibility defined by:

$$
G\left(\mathbf{k}, q_{z}\right)=\left\langle\mathbf{S}_{\mathbf{k}, q_{z}} \cdot \mathbf{S}_{-\mathbf{k},-q_{z}}\right\rangle,
$$

with the statistical average:

$$
\begin{aligned}
\langle\ldots\rangle= & \frac{1}{Z} \int_{-\infty}^{+\infty} \prod_{i, \ell} d^{2} \mathbf{S}_{\ell}\left(\mathbf{r}_{i}\right) \\
& \times \delta\left[\sum_{i, \ell} \mathbf{S}_{\ell}^{2}\left(\mathbf{r}_{i}\right)-N\right] \ldots e^{-\beta H[\mathbf{S}]} .
\end{aligned}
$$

From Eqs. (10) and (14) we obtain the critical temperature in the form:

$$
\beta_{c}=\lim _{N \rightarrow \infty} \frac{2}{N} \sum_{\mathbf{k}, q_{z}} \frac{1}{J_{0}-J\left(\mathbf{k}, q_{z}\right)} .
$$

For one (1D) and two-dimensional (2D) systems with short-range interactions the ordered phase exists only in the zero temperature limit, hence for dimensions $d \leq$ 2 the spherical model does not exhibit phase transition with $T_{c}>0$, in agreement with Mermin-Wagner theorem ${ }^{25}$ For $d=3$ the critical temperature is finite

$$
\frac{2 k_{B} T_{c}}{J_{0}} \approx 0.659
$$

where the factor 2 in front of $k_{B} T_{c}$ comes from the number of components of the vector XY model ${ }^{25}$

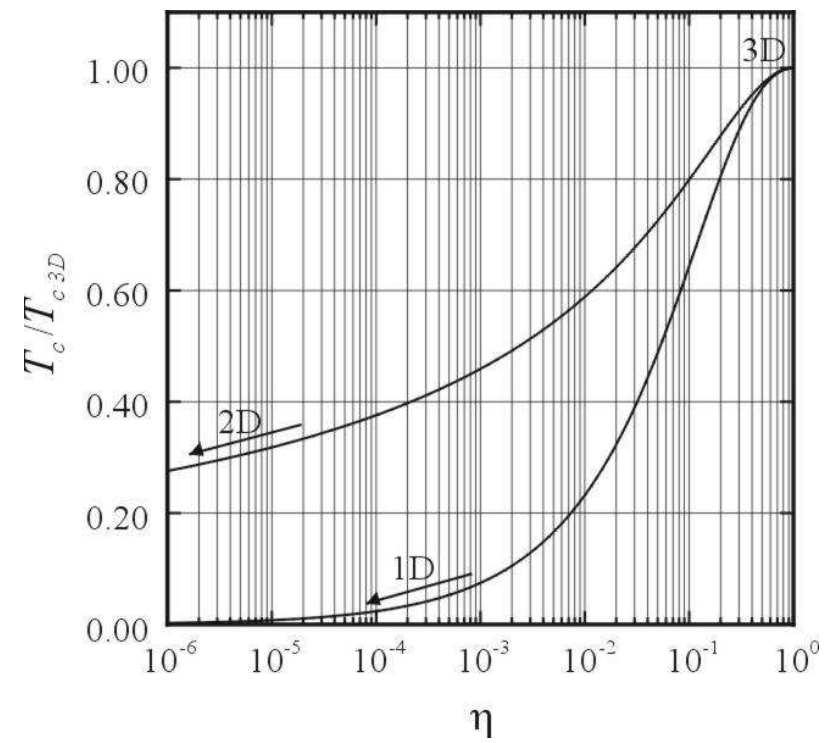

Figure 2: Superconducting critical temperature $T_{c}$ as a function of anisotropy $\eta$ for crossovers from isotropic 3D to $1 \mathrm{D}$ or $2 \mathrm{D}$ anisotropic systems in logarithmic scale for $\eta$.

Introducing spatial anisotropy parameter $0 \leq \eta \leq$ 1 (being the ratio between $J_{\|}$and $\left.J_{\perp}\right), J\left(\mathbf{k}, q_{z}\right)$ can be written in a form to accommodate $3 \mathrm{D} \rightarrow 2 \mathrm{D}$ crossover from the isotropic superconductor on the threedimensional cubic lattice $(\eta=1)$ to the uncoupled $(\eta=0)$ copper-oxide planes (see, Fig. 2):

$$
\begin{gathered}
J\left(\mathbf{k}, q_{z}\right)=2 J\left[\cos \left(a k_{x}\right)+\cos \left(a k_{y}\right)+\eta \cos \left(c q_{z}\right)\right] \\
J \equiv J_{\|}, \quad \eta=J_{\perp} / J_{\|}
\end{gathered}
$$

In a similar way we can study a $3 \mathrm{D} \rightarrow 1 \mathrm{D}$ crossover from the isotropic superconductor to the uncoupled onedimensional chains:

$$
\begin{gathered}
J\left(\mathbf{k}, q_{z}\right)=2 J\left\{\eta\left[\cos \left(a k_{x}\right)+\cos \left(a k_{y}\right)\right]+\cos \left(c q_{z}\right)\right\} \\
J \equiv J_{\perp}, \quad \eta=J_{\|} / J_{\perp} .
\end{gathered}
$$

The critical temperature can be found for both cases from Eq. [19] by performing momentum integration. In the thermodynamic limit we have:

$$
\lim _{N \rightarrow \infty} \frac{1}{N} \sum_{\mathbf{k}, q_{z}} \cdots=\int_{-\frac{\pi}{a}}^{\frac{\pi}{a}} \frac{d^{2} \mathbf{k}}{(2 \pi / a)^{2}} \int_{-\frac{\pi}{c}}^{\frac{\pi}{c}} \frac{d q_{z}}{(2 \pi / c)} \ldots
$$

For $3 \mathrm{D} \rightarrow 2 \mathrm{D}$ crossover it reads:

$$
\begin{aligned}
& \frac{J_{0}}{k_{B} T_{c}(\eta)}=\frac{2(2+\eta)(2 \sqrt{1+\eta}-\sqrt{4+2 \eta})}{\eta \pi^{2}} \\
& \times \mathbf{K}\left[\frac{1}{2 \eta}(2 \sqrt{1+\eta}-\sqrt{4+2 \eta})(\sqrt{4+2 \eta}+2)\right] \\
& \times \mathbf{K}\left[\frac{1}{2 \eta}(2 \sqrt{1+\eta}-\sqrt{4+2 \eta})(\sqrt{4+2 \eta}-2)\right],
\end{aligned}
$$


while for $3 \mathrm{D} \rightarrow 1 \mathrm{D}$ changeover the Eq. (19):

$$
\begin{aligned}
& \frac{J_{0}}{k_{B} T_{c}(\eta)}=\frac{2(1+2 \eta)(2 \sqrt{1+\eta}-\sqrt{2-4 \eta})}{\pi^{2} \sqrt{\eta}} \\
& \times \mathbf{K}\left[2\left(\sqrt{\eta}-\sqrt{\frac{1}{2}+\eta}\right)\left(\sqrt{\frac{1}{2}+\eta}-\sqrt{1+\eta}\right)\right] \\
& \times \mathbf{K}\left[2\left(\sqrt{\eta}+\sqrt{\frac{1}{2}+\eta}\right)\left(\sqrt{\frac{1}{2}+\eta}-\sqrt{1+\eta}\right)\right],
\end{aligned}
$$

where $\mathbf{K}(x)$ is the complete elliptic integral of the first kind ${ }^{27}$ It is interesting, how these $T_{c}(\eta)$ values behave in the limiting cases. For the $3 \mathrm{D} \rightarrow 2 \mathrm{D}$ crossover from Eq. (24) we obtain:

$$
\frac{k_{B} T_{c}}{J_{0}} \approx\left\{\begin{array}{l}
1.319-0.0944 \eta^{2}-0.0559 \eta^{4} \text { for } \eta \rightarrow 1 \\
\frac{2 \pi}{\ln (32 / \eta)}-\frac{\pi \eta[\ln (32 / \eta)+2]}{2 \ln ^{2}(32 / \eta)} \text { for } \eta \rightarrow 0,
\end{array}\right.
$$

while for $3 \mathrm{D} \rightarrow 1 \mathrm{D}$ changeover, Eq. (25) it follows that:

$$
\frac{k_{B} T_{c}}{J_{0}} \approx\left\{\begin{array}{l}
1.319-0.0944 \eta^{2}-0.106 \eta^{4} \text { for } \eta \rightarrow 1 \\
\frac{(2+\sqrt{2}) \pi^{2} \sqrt{\eta}}{4 \mathbf{K}^{2}(3-2 \sqrt{2})} \text { for } \eta \rightarrow 0 .
\end{array}\right.
$$

The results are presented in Fig. 2 2 and in Table [ When the in-plane interactions are reduced, the critical temperature drops quite rapidly as a function of $\eta$ compared to the isotropic system and for $\eta=10^{-5}$ is less than $1 \%$ of the isotropic value. However, in the case of the reduced coupling along $c$-axis (weakly coupled planes), even for anisotropy parameter as small as $\eta=10^{-6}$ the critical temperature is still about $30 \%$ of the isotropic three-dimensional value. This result is very important as it proves that even very small inter-planar coupling (sometimes too small to be directly observed experimentally) renders the phase transition in the $3 \mathrm{D}$ universality class $\stackrel{14}{=}$ with the critical temperature decreasing slowly like $T_{c} \sim 1 / \log \eta$. This result raises serious doubts whether the outcome of intercalation experiments in cuprates, 15 which show small changes of the critical temperature with increase of the inter-layer distance can be interpreted as an argument in favor for a purely 2D nature of high- $T_{c}$ superconductivity.

\section{MULTI LAYERED SYSTEM}

To describe superconducting cuprates of homologous families, we modify the model presented in Sec. [I] to include the presence of the $n$-layer block structure. To this end, we group every $n$ consecutive layers in blocks. The intra-layer (in the $a b$ planes) interactions are given by $J_{\|}>0$, while the plane coupling along $c$ direction is different inside $J_{\perp}>0$ and between $J_{\perp}^{\prime}>0$ blocks,

\begin{tabular}{|c|c|c|}
\hline$\eta$ & $3 \mathrm{D} \rightarrow 2 \mathrm{D}: \frac{T_{c}(\eta)}{T_{c}(1)}$ & $3 \mathrm{D} \rightarrow 1 \mathrm{D}: \frac{T_{c}(\eta)}{T_{c}(1)}$ \\
\hline \hline 1 & 1 & 1 \\
\hline 0.1 & 0.80 & 0.64 \\
\hline 0.01 & 0.59 & 0.23 \\
\hline 0.001 & 0.46 & 0.074 \\
\hline 0.0001 & 0.38 & 0.024 \\
\hline 0.00001 & 0.32 & 0.0075 \\
\hline 0.000001 & 0.28 & 0.0024 \\
\hline
\end{tabular}

Table I: Ratio of the superconducting critical temperature $T_{c}(\eta)$ to the isotropic three-dimensional value $T_{c}(1)$ for several values of the anisotropy parameter $\eta$ facilitating the crossovers from isotropic $3 \mathrm{D}$ to anisotropic $1 \mathrm{D}$ or $2 \mathrm{D}$ systems.

respectively (see, Fig. 3). The phase Hamiltonian of the system can now be written:

$$
\begin{aligned}
H & =-J_{\|} \sum_{\ell} \sum_{i<j} \cos \left[\varphi_{\ell}\left(\mathbf{r}_{i}\right)-\varphi_{\ell}\left(\mathbf{r}_{j}\right)\right] \\
& -J_{\perp} \sum_{i} \sum_{m} \sum_{\alpha \geq 0}^{n-2} \cos \left[\varphi_{m n+\alpha}\left(\mathbf{r}_{i}\right)-\varphi_{m n+\alpha+1}\left(\mathbf{r}_{i}\right)\right] \\
& -J_{\perp}^{\prime} \sum_{i} \sum_{m} \cos \left[\varphi_{m n+n-1}\left(\mathbf{r}_{i}\right)-\varphi_{m n+n}\left(\mathbf{r}_{i}\right)\right],(28)
\end{aligned}
$$

where indices $i$ and $j$ run over sites in the $a b$-planes of the $\ell$-th layer, while $\alpha$ numbers the layers within the $m$ th block. Introducing two-dimensional vectors from Eq. (3), the Hamiltonian reads:

$$
\begin{aligned}
H= & -J_{\|} \sum_{\ell} \sum_{i<j} \mathbf{S}_{\ell}\left(\mathbf{r}_{i}\right) \mathbf{S}_{\ell}\left(\mathbf{r}_{j}\right) \\
& -J_{\perp} \sum_{i} \sum_{m} \sum_{\alpha \geq 0}^{n-2} \mathbf{S}_{m n+\alpha}\left(\mathbf{r}_{i}\right) \mathbf{S}_{m n+\alpha+1}\left(\mathbf{r}_{i}\right) \\
& -J_{\perp}^{\prime} \sum_{i} \sum_{m} \mathbf{S}_{m n+n-1}\left(\mathbf{r}_{i}\right) \mathbf{S}_{m n+n}\left(\mathbf{r}_{i}\right) .
\end{aligned}
$$

Furthermore, we make use of the spherical closure relation in Eq. (6). However, the direct implementation of the Eq. (6) is obstructed by the lack of complete translational symmetry: grouping of layers within blocks breaks translational symmetry along $c$ axis, since the inter-plane coupling varies with period of $n$, when moving from one plane to another. As a result, the three-dimensional Fourier transform of the $\mathbf{S}_{\ell}\left(\mathbf{r}_{i}\right)$ variables in Eq. (11), used to diagonalize single-layer Hamiltonian in the previous Section, cannot be performed. To overcome this difficulty, we implement a combination of two-dimensional Fourier transform for in-plane vector variables

$$
\mathbf{S}_{\ell}\left(\mathbf{r}_{i}\right)=\frac{1}{N_{\|}} \sum_{\mathbf{k}} \mathbf{S}_{\mathbf{k} \ell} e^{-i \mathbf{k r}_{i}} .
$$

and transfer matrix method for one-dimensional decorated structure along $c$-axis $\underset{28}{28}$ This operation diagonalizes all terms in the Hamiltonian in Eq. (29) with respect 


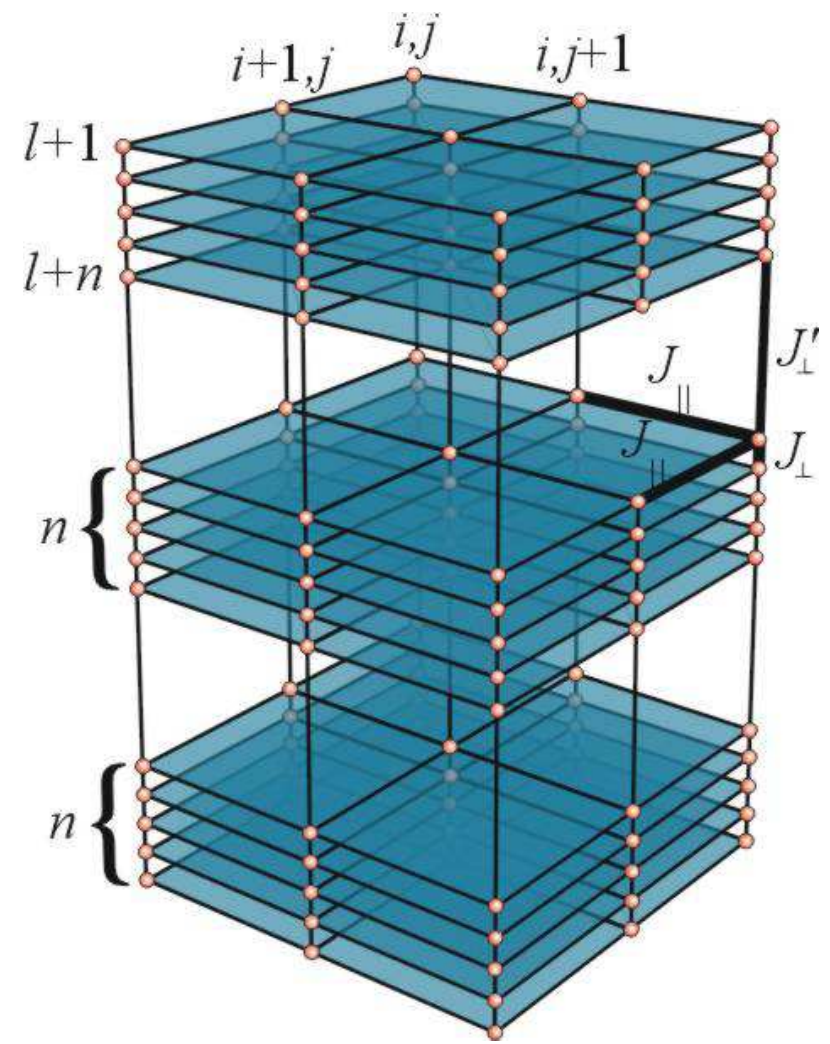

Figure 3: (Color online) Structure of the multi-layer high-temperature superconductors of homologous series of cuprates. Coupled $\mathrm{Cu}-\mathrm{O}$ planes are grouped in blocks of $n$ planes. The microscopic phase stiffnesses $J_{\|}, J_{\perp}$ and $J_{\perp}^{\prime}$ are indicated by the thick lines. to $\mathbf{k}$, leaving the dependence on layer index $\ell$ unchanged. Therefore, the partition function can be written in the form:

$$
\begin{aligned}
Z= & \int_{-\infty}^{+\infty} \frac{d \zeta}{2 \pi i} \exp \left\{N \zeta+\frac{1}{2} \ln \int_{-\infty}^{+\infty} \prod_{\mathbf{k}, \ell} d^{2} \mathbf{S}_{\mathbf{k} \ell}\right. \\
& \left.\times \exp \left[-\frac{1}{N_{\|}} \sum_{\mathbf{k}, \ell, \ell^{\prime}} \mathbf{S}_{\mathbf{k} \ell} A_{N_{\perp}}^{\ell \ell^{\prime}}(\mathbf{k}) \mathbf{S}_{\mathbf{k} \ell^{\prime}}\right]\right\},
\end{aligned}
$$

where $A_{N_{\perp}}^{\ell \ell^{\prime}}(\mathbf{k})$ is an element of a square $N_{\perp} \times N_{\perp}$ band matrix, appearing as a result of non-trivial coupling structure along $c$-direction:

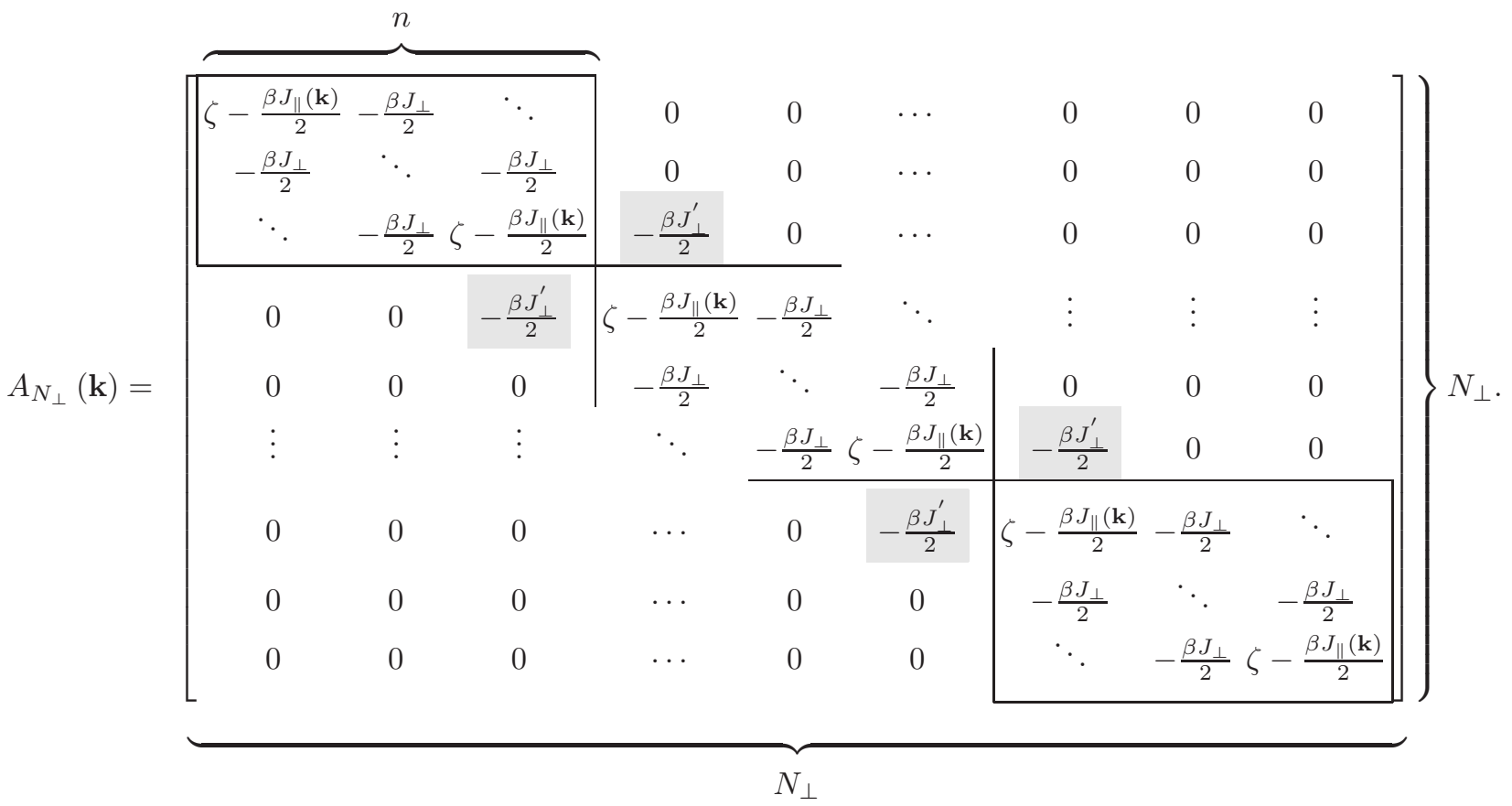


where

$$
J_{\|}(\mathbf{k})=2 J_{\|}\left[\cos \left(a k_{x}\right)+\cos \left(a k_{y}\right)\right] .
$$

Here, $A_{N_{\perp}}$ has square $n \times n$ diagonal blocks:

$$
B_{n}=\left[\begin{array}{cccc}
\zeta-\frac{\beta J_{\|}(\mathbf{k})}{2} & -\frac{\beta J_{\perp}}{2} & 0 & 0 \\
-\frac{\beta J_{\perp}}{2} & \ddots & \ddots & 0 \\
0 & \ddots & \ddots & -\frac{\beta J_{\perp}}{\beta J_{\|}(\mathbf{k})} \\
0 & 0 & -\frac{\beta J_{\perp}}{2} & \zeta-\frac{1}{2}
\end{array}\right]
$$

containing interactions inside the stack of $n$ planes. Performing Gaussian integration in Eq. (31), one arrives at the equation involving determinant of the matrix in Eq. (32):

$$
Z=\int_{-\infty}^{+\infty} \frac{d \zeta}{2 \pi i} \exp \left[N \zeta+\sum_{\mathbf{k}} \ln \left(\frac{N_{\|}^{\frac{1}{2} N_{\perp}}}{\operatorname{det} A_{N_{\perp}}}\right)\right] .
$$

Thus, the problem of finding the partition function in Eq. (35) is to find a way to calculate the determinant of the matrix $A_{N_{\perp}}$ :

$$
\operatorname{det}\left(A_{N_{\perp}}\right) \equiv\left|A_{N_{\perp}}\right| .
$$

However, because of the band structure of the matrix $A_{N_{\perp}}$ it can be conveniently done using generalised Laplace method. As a result, the determinant in Eq. (36) can be written in a recurrence form:

$$
\left|A_{N_{\perp}}\right|=\left|B_{n}\right|\left|A_{N_{\perp}-n}\right|-\left(\frac{\beta J_{\perp}^{\prime}}{2}\right)^{2}\left|B_{n-1}\right|\left|A_{N_{\perp}-n-1}\right|,
$$

where $A_{N_{\perp}-n}$ and $A_{N_{\perp-n-1}}$ arise from removing the first $n$ and $n+1$ rows and columns of $A_{N_{\perp}}$, respectively. Similarly to Eq. (37), for $\left|A_{N_{\perp}-1}\right|$ the recurrence formula can be written as:

$$
\begin{aligned}
\left|A_{N_{\perp}-1}\right| & =\left|B_{n-1}\right|\left|A_{N_{\perp}-n}\right| \\
& -\left(\frac{\beta J_{\perp}^{\prime}}{2}\right)^{2}\left|B_{n-2}\right|\left|A_{N_{\perp}-n-1}\right| .
\end{aligned}
$$

Defining $\left|B_{0}\right|=1$, the formula in Eq. (38) holds for any $n \geq 2$. The results for the single-layer system $(n=1)$ from the previous Section can be recovered by performing calculations for any $n \geq 2$ and setting $J_{\perp}=J_{\perp}^{\prime}$. Using Eqs. (37) and (38) we can write the recurrence relation for the $A_{N_{\perp}}$ in Eq. (32) as an operator equation:

$$
\left[\begin{array}{c}
\left|A_{N_{\perp}}\right| \\
\left|A_{N_{\perp}-1}\right|
\end{array}\right]=\mathbf{T}_{1}\left[\begin{array}{c}
\left|A_{N_{\perp}-n}\right| \\
\left|A_{N_{\perp}-n-1}\right|
\end{array}\right]
$$

where $\mathbf{T}_{1}$ is transfer matrix:

$$
\mathbf{T}_{1}=\left[\begin{array}{cc}
\left|B_{n}\right| & -\left(\frac{\beta J_{\perp}^{\prime}}{2}\right)^{2}\left|B_{n-1}\right| \\
\left|B_{n-1}\right| & -\left(\frac{\beta J_{\perp}^{\prime}}{2}\right)^{2}\left|B_{n-2}\right|
\end{array}\right] .
$$

Applying the rule in Eq. (39) recursively, one obtains:

$$
\left[\begin{array}{c}
\left|A_{N_{\perp}}\right| \\
\left|A_{N_{\perp}-1}\right|
\end{array}\right]=\mathbf{T}_{1}^{\frac{1}{n} N_{\perp}-1}\left[\begin{array}{c}
\left|A_{n}\right| \\
\left|A_{n-1}\right|
\end{array}\right] .
$$

Therefore, finding the solution of the Eq. (41) reduces to the problem of determining the power of the transfer matrix operator $\mathbf{T}_{1}$. To this end we diagonalize the matrix $\mathbf{T}_{1}$ with the help of a unitary transformation $\mathcal{U}_{1}$ (see, Appendix @):

$$
\mathbf{T}_{1}=\mathcal{U}_{1}\left[\begin{array}{cc}
\lambda_{1} & 0 \\
0 & \lambda_{2}
\end{array}\right] \mathcal{U}_{1}^{-1}
$$

where $\lambda_{1}$ and $\lambda_{2}$ are eigenvalues of $\mathbf{T}_{1}$, whereas $\mathcal{U}_{1}$ is a unitary matrix built of eigenvectors of $\mathbf{T}_{1}$. Therefore,

$$
\mathbf{T}_{1}^{\frac{1}{n} N_{\perp}-1}=\mathcal{U}_{1}\left[\begin{array}{cc}
\lambda_{1}^{\frac{1}{n} N_{\perp}-1} & 0 \\
0 & \lambda_{2}^{\frac{1}{n} N_{\perp}-1}
\end{array}\right] \mathcal{U}_{1}^{-1}
$$

The eigenvalue $\lambda_{1}$ is chosen to satisfy $\lambda_{1}>\lambda_{2}$. In the large- $N_{\perp}$ (thermodynamic) limit only the largest eigenvalue $\lambda_{1}$ will contribute to the statistical sum in Eq. (35),

$$
\begin{aligned}
{\left[\begin{array}{cc}
\lambda_{1} & 0 \\
0 & \lambda_{2}
\end{array}\right]^{\frac{1}{n} N_{\perp}-1} } & =\left\{\lambda_{1}\left[\begin{array}{cc}
1 & 0 \\
0 & \lambda_{2} / \lambda_{1}
\end{array}\right]\right\}^{\frac{1}{n} N_{\perp}-1} \\
& =\lambda_{1}^{\frac{1}{n} N_{\perp}-1}\left[\begin{array}{ll}
1 & 0 \\
0 & 0
\end{array}\right]
\end{aligned}
$$

Combining Eqs. (41), (43) and (44) we obtain finally:

$$
\left[\begin{array}{c}
\left|A_{N_{\perp}}\right| \\
\left|A_{N_{\perp}-1}\right|
\end{array}\right] \underset{N_{\perp} \rightarrow \infty}{=} \lambda_{1}^{\frac{1}{n} N_{\perp}-1} \mathcal{U}_{1}\left[\begin{array}{ll}
1 & 0 \\
0 & 0
\end{array}\right] \mathcal{U}_{1}^{-1}\left[\begin{array}{c}
\left|A_{n}\right| \\
\left|A_{n-1}\right|
\end{array}\right]
$$

where: 


$$
\lambda_{1}=\frac{1}{2}\left\{\left|B_{n}\right|-\left(\frac{\beta J_{\perp}^{\prime}}{2}\right)^{2}\left|B_{n-2}\right|+\sqrt{\left[\left|B_{n}\right|+\left(\frac{\beta J_{\perp}^{\prime}}{2}\right)^{2}\left|B_{n-2}\right|\right]^{2}-\left(\beta J_{\perp}^{\prime}\right)^{2}\left|B_{n-1}\right|^{2}}\right\}
$$

From Eq. 450 one can extract the value of determinant of the matrix $A_{N_{\perp}}$ :

$$
\left|A_{N_{\perp}}\right|=\lambda_{1}^{\frac{1}{n} N_{\perp}-1}(\mathcal{U})_{11}\left[\left(\mathcal{U}_{1}^{-1}\right)_{11}\left|B_{n}\right|+\left(\mathcal{U}_{1}^{-1}\right)_{21}\left|B_{n-1}\right|\right]
$$

where $(\mathcal{U})_{\mu \nu}$ and $\left(\mathcal{U}_{1}^{-1}\right)_{\mu \nu}$ denote elements of the matrix $\mathcal{U}_{1}$ and the inverse matrix $\mathcal{U}_{1}^{-1}$, respectively (see, Appendix A). Explicitly,

$$
\left|A_{N_{\perp}}\right|=\mathcal{C}\left[\frac{\left|B_{n}\right|-\left(\frac{\beta J_{\perp}^{\prime}}{2}\right)^{2}\left|B_{n-2}\right|}{2}+\frac{\sqrt{\left[\left|B_{n}\right|+\left(\frac{\beta J_{\perp}^{\prime}}{2}\right)^{2}\left|B_{n-2}\right|\right]^{2}-\left(\beta J_{\perp}^{\prime}\right)^{2}\left|B_{n-1}\right|^{2}}}{2}\right]^{\frac{1}{n} N_{\perp}-1}
$$

is the function of determinants of matrices $B_{n}$ with intrablock interactions $J_{\perp}$ and

$$
\mathcal{C}=\left(\mathcal{U}_{1}\right)_{11}\left[\left(\mathcal{U}_{1}^{-1}\right)_{11}\left|B_{n}\right|+\left(\mathcal{U}_{1}^{-1}\right)_{21}\left|B_{n-1}\right|\right]
$$

The determinant of the matrix $B_{n}$ in Eq. (34) can be found using similar method by employing the Laplace expansion (see Eq. (37)- (45)). However, since the order of $B_{n}$ is finite, both eigenvalues of the new transfer matrix $\mathbf{T}_{2}$ must be taken into account:

$$
\begin{gathered}
{\left[\begin{array}{c}
\left|B_{n+1}\right| \\
\left|B_{n}\right|
\end{array}\right]=\mathbf{T}_{2}^{n / 2-1}\left[\begin{array}{l}
\left|B_{2}\right| \\
\left|B_{1}\right|
\end{array}\right]=\mathcal{U}_{2}} \\
\times\left[\begin{array}{cc}
\lambda_{+}^{n / 2-1} & 0 \\
0 & \lambda_{-}^{n / 2-1}
\end{array}\right] \mathcal{U}_{2}^{-1}\left[\begin{array}{l}
\left|B_{2}\right| \\
\left|B_{1}\right|
\end{array}\right]
\end{gathered}
$$

where transfer matrix $\mathbf{T}_{2}$ is:

$$
\mathbf{T}_{2}=\left[\begin{array}{ll}
{\left[\zeta-\frac{\beta J_{\|}(\mathbf{k})}{2}\right]^{2}-\frac{\beta^{2} J_{\perp}^{2}}{4}} & -\frac{\beta^{2} J_{\perp}^{2}}{4}\left[\zeta-\frac{\beta J_{\|}(\mathbf{k})}{2}\right] \\
\zeta-\frac{\beta J_{\|}(\mathbf{k})}{2} & -\frac{\beta^{2} J_{\perp}^{2}}{4}
\end{array}\right],
$$

$\mathcal{U}_{2}$ is matrix built of eigenvectors of $\mathbf{T}_{2}$ and eigenvalues $\lambda_{+}, \lambda_{-}$of $\mathbf{T}_{2}$ and read:

$$
\begin{aligned}
\lambda_{ \pm} & =\frac{1}{2}\left\{\left[\zeta-\frac{\beta J_{\|}(\mathbf{k})}{2}\right]^{2}-\frac{\beta^{2} J_{\perp}^{2}}{2} \pm\left[\zeta-\frac{\beta J_{\|}(\mathbf{k})}{2}\right]\right. \\
& \left.\times \sqrt{\left[\zeta-\frac{\beta J_{\|}(\mathbf{k})}{2}\right]^{2}-\left(\beta J_{\perp}\right)^{2}}\right\} .
\end{aligned}
$$

Finally, we arrive at the value of $\left|B_{n}\right|$ determinant:

$$
\begin{aligned}
\left|B_{n}\right| & =\frac{\left|B_{2}\right|}{2}\left(\lambda_{+}^{\frac{n}{2}}+\lambda_{-}^{\frac{n}{2}}\right)+\frac{\left|B_{1}\right|}{2}\left(\lambda_{+}^{\frac{n}{2}}-\lambda_{-}^{\frac{n}{2}}\right) \\
& \times \frac{2\left|B_{2}\right|-\left(\beta J_{\perp}\right)^{2}}{\sqrt{\left[\zeta-\frac{\beta J_{\|}(\mathbf{k})}{2}\right]^{2}-\left(\beta J_{\perp}\right)^{2}}} .
\end{aligned}
$$

with $\lambda_{+}$and $\lambda_{-}$given by Eq. (52). Thus, with the result in Eq. (53), the calculation of the determinant of the matrix $A_{N_{\perp}}$ in Eq. (48) and thereby the partition function given by Eq. (35) is completed.

\section{CRITICAL TEMPERATURE}

Using the partition function in Eq. (35) with the definitions in Eqs. (46) and (49) the free energy $f$ is given by:

$$
\begin{aligned}
& f=\frac{\zeta}{\beta}-\frac{1}{\beta N_{\perp} N_{\|}} \sum_{\mathbf{k}} \ln \lambda_{1}^{\frac{1}{n} N_{\perp}-1}(\mathbf{k})+\frac{1}{2 \beta N_{\|}} \ln N_{\|} \\
& -\frac{1}{\beta N_{\perp} N_{\|}} \sum_{\mathbf{k}} \ln \mathcal{C}
\end{aligned}
$$

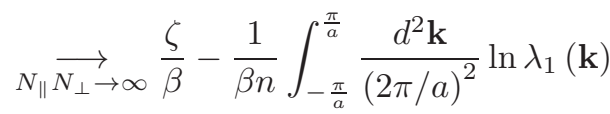

while the saddle-point equation for the spherical Lagrange multiplier becomes:

$$
\left.\frac{\partial f(\zeta)}{\partial \zeta}\right|_{\zeta=\zeta_{0}}=0
$$


Explicitly,

$$
1=\left.\frac{1}{n} \int_{-\frac{\pi}{a}}^{\frac{\pi}{a}} \frac{d^{2} \mathbf{k}}{(2 \pi / a)^{2}} \frac{\frac{\partial C_{n}(\mathbf{k})}{\partial \zeta} \sqrt{D_{n}(\mathbf{k})}+\frac{1}{2} \frac{\partial D_{n}(\mathbf{k})}{\partial \zeta}}{C_{n}(\mathbf{k}) \sqrt{D_{n}(\mathbf{k})}+D_{n}(\mathbf{k})}\right|_{\zeta=\zeta_{0}},
$$

where we have defined:

$$
\begin{aligned}
C_{n}(\mathbf{k}) & =\left|B_{n}\right|-\left(\frac{\beta J_{\perp}^{\prime}}{2}\right)^{2}\left|B_{n-2}\right| \\
D_{n}(\mathbf{k}) & =\left[\left|B_{n}\right|+\left(\frac{\beta J_{\perp}^{\prime}}{2}\right)^{2}\left|B_{n-2}\right|\right]^{2} \\
& -\left(\beta J_{\perp}^{\prime}\right)^{2}\left|B_{n-1}\right|^{2} .
\end{aligned}
$$

The in-plane momentum dependence of $\lambda_{1}(\mathbf{k}), C_{n}(\mathbf{k})$ and $D_{n}(\mathbf{k})$ comes from $J_{\| \mid}(\mathbf{k})$ dependence of $\left|B_{n}\right|$, see Eq. (53). The saddle-point value $\zeta_{0}$ can be determined from the condition that in criticality order-parameter susceptibility becomes infinite (see, Eq. (15)):

$$
\left.\frac{C_{n}(\mathbf{k}) \sqrt{D_{n}(\mathbf{k})}+D_{n}(\mathbf{k})}{\frac{\partial C_{n}(\mathbf{k})}{\partial \zeta} \sqrt{D_{n}(\mathbf{k})}+\frac{1}{2} \frac{\partial D_{n}(\mathbf{k})}{\partial \zeta}}\right|_{\begin{array}{l}
\mathbf{k}=0 \\
\zeta=\zeta_{0}
\end{array}}=0 .
$$

Closed formulas for the critical temperature can be easily obtained only for small values of $n$. For example, in the case of a bilayer system $(n=2)$, the respective determinants read:

$$
\begin{aligned}
& \left|B_{2}\right|=\left[\zeta-\frac{\beta J_{\|}(\mathbf{k})}{2}\right]^{2}-\left(\frac{\beta J_{\perp}}{2}\right)^{2}, \\
& \left|B_{1}\right|=\zeta-\frac{\beta J_{\|}(\mathbf{k})}{2} \\
& \left|B_{0}\right|=1
\end{aligned}
$$

and the saddle point equation in Eq. (56) can be written explicitly:

$$
\begin{aligned}
1= & \int_{-\frac{\pi}{a}}^{\frac{\pi}{a}} \frac{d^{2} \mathbf{k}}{(2 \pi / a)^{2}} \frac{\zeta-\frac{1}{2} \beta J_{\|}(\mathbf{k})}{\sqrt{\left[\zeta_{0}-\frac{\beta}{2}\left(J_{\|}(\mathbf{k})-J_{\perp}^{\prime}\right)\right]^{2}-\frac{\beta^{2} J_{\perp}^{2}}{4}}} \\
& \times \frac{1}{\sqrt{\left[\zeta_{0}-\frac{\beta}{2}\left(J_{\|}(\mathbf{k})+J_{\perp}^{\prime}\right)\right]^{2}-\frac{\beta^{2} J_{\perp}^{2}}{4}}}
\end{aligned}
$$

with the Lagrange multiplier:

$$
\zeta_{0}=\frac{\beta}{2}\left[J_{\|}(\mathbf{k}=0)+J_{\perp}+J_{\perp}^{\prime}\right] .
$$

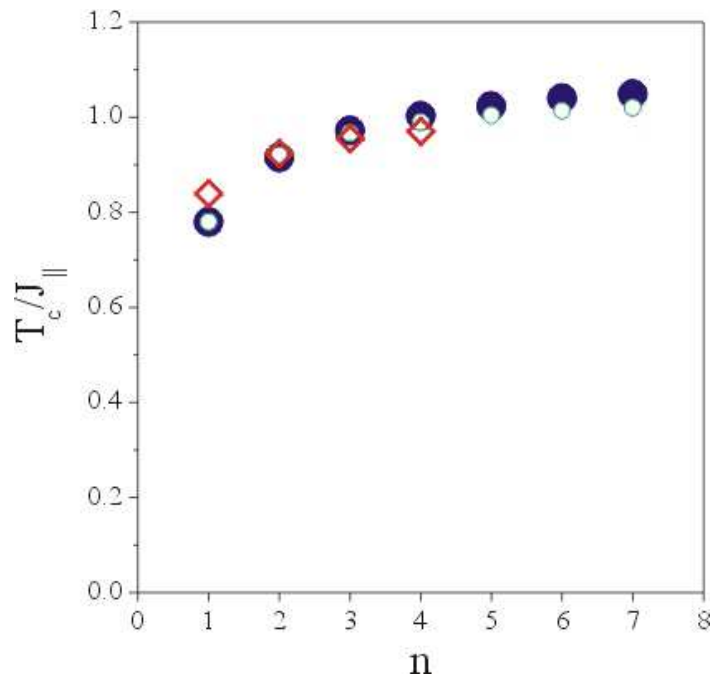

Figure 4: (Color online) Critical temperature vs. number of $\mathrm{Cu}-\mathrm{O}$ layers within block for $J_{\perp} / J_{\|}=0.1$ and $J_{\perp}^{\prime} / J_{\|}=0.01$ (blue filled circles). Empty circles (green) denote the critical temperature dependence $T_{c}(n)=T_{c}(1)+0.28(1-1 / n)$, from Ref. 20 (factor of 0.28 was chosen to fit the results of the present paper). Diamonds (red) represent results from MonteCarlo (MC) simulations of the classical XY model (Carlson, et al., Ref. 29). The data of Ref. 29 is scaled by overall factor of 0.77 to accommodate the finite size effect of MC simulations.

Finally, the critical temperature of this system (cf. Eq. (19)) is given by

$$
\begin{aligned}
\beta_{c}= & \frac{1}{2} \int_{-\infty}^{+\infty} d \xi \rho(\xi) \frac{2(2-\xi) J_{\|}+J_{\perp}+J_{\perp}^{\prime}}{\sqrt{(2-\xi) J_{\|}\left[(2-\xi) J_{\|}+J_{\perp}\right]}} \\
& \times \frac{1}{\sqrt{\left[2(2-\xi) J_{\|}+\left(J_{\perp}+2 J_{\perp}^{\prime}\right)\right]^{2}-J_{\perp}^{2}}}
\end{aligned}
$$

with

$$
\rho(\xi)=\int_{-\frac{\pi}{a}}^{\frac{\pi}{a}} \frac{d^{2} \mathbf{k}}{(2 \pi / a)^{2}} \delta\left[\xi-\cos \left(a k_{x}\right)-\cos \left(a k_{y}\right)\right]
$$

being density of states of the square lattice given by:

$$
\rho(\xi)=\frac{1}{\pi^{2}} \mathbf{K}\left(\sqrt{1-\frac{\xi^{2}}{4}}\right) \Theta\left(1-\frac{|\xi|}{2}\right),
$$

where $\Theta(x)$ is the unit-step function. For higher values of $n$ we can resort to direct numerical evaluation of Eqs. (56) and (58) in order to compute $T_{c}(n)$.

The dependence of the critical temperature on the number of layers $n$ in a block was presented in Fig. 4 Phase stiffnesses $J_{\|}, J_{\perp}$ and $J_{\perp}^{\prime}$ were chosen to satisfy

$$
J_{\|} \gg J_{\perp}>J_{\perp}^{\prime},
$$

which is physically reasonable, since the interlayer couplings $J_{\perp}$ and $J_{\perp}^{\prime}$ are much smaller than in-plane phase 


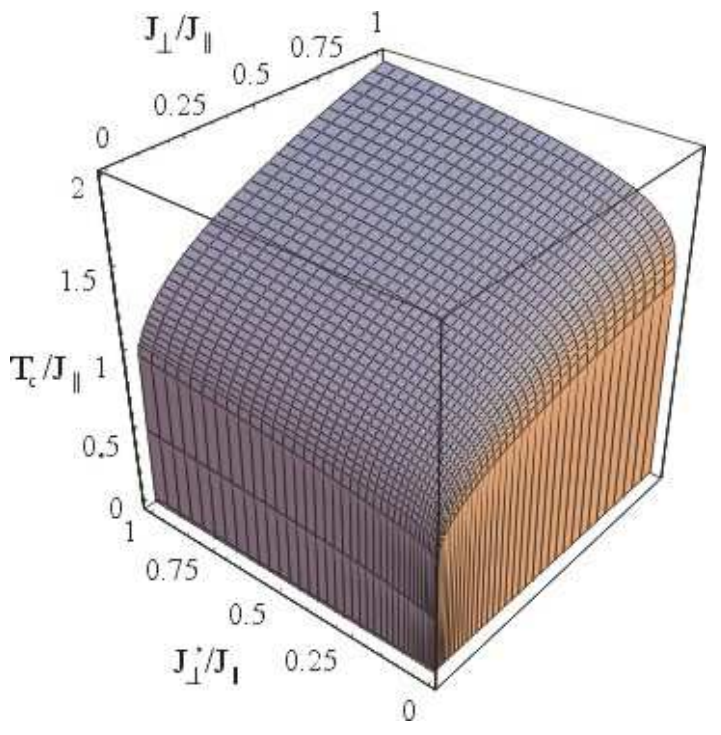

Figure 5: (Color online) Critical temperature $T_{c}(n)$ for $n=4$ as a function of $c$-axis phase stiffnesses $J_{\perp}$ and $J_{\perp}^{\prime}$.

stiffness $J_{\|}$and inter-layer (intra-block) phase stiffness $J_{\perp}$ is greater than inter-block coupling $J_{\perp}^{\prime}$. We found that the critical temperature increases monotonically with $n$. This has a simple explanation: for $n=1$ (singlelayer system) with the fixed phase stiffness $J_{\|}$, the critical temperature is determined by $J_{\perp}^{\prime}$, see Fig. 2 On the other hand, in the limit of $n \rightarrow \infty, n$-layer blocks are of infinite size and inter-block coupling $J_{\perp}^{\prime}$ is effectively replaced by $J_{\perp}$. Because $J_{\perp}>J_{\perp}^{\prime}$, according to the result from the previous Section, $T_{c}(n=\infty)>T_{c}(n=1)$. For intermediate values on $n$, one can expect a monotonic increase of the $T_{c}(n)$ with increasing $n$, as depicted in Fig. 44 In this respect, our findings are similar to the results from Ref. 20, where the increase of the critical temperature of identically doped members of the same homologous series due to the interlayer Coulomb interaction was found to be

$$
T_{c}(n)=T_{c}(1)+\text { const } \times\left(1-\frac{1}{n}\right) .
$$

Similar results were obtained by Monte-Carlo simulations $\stackrel{29}{\underline{n}}$ where $T_{c}(n)$ was computed numerically by means of the Binder parameter ${ }^{30}$ for systems of size up to $24 \times 24 \times 24$. Additionally, we present the influence of inter-planar coupling constants $J_{\perp}$ and $J_{\perp}^{\prime}$ on $T_{c}$, see Figs. [5 and [6] For a bilayer system the role of both interaction constants is identical. For systems with $n \geq 3$ the influence of the inter-block coupling $J_{\perp}^{\prime}$ is less visible, however $J_{\perp}^{\prime}=0$ suppresses the critical temperature to zero, since for finite $n$ the isolated stack of $n$ coupled layers is effectively quasi two-dimensional. Therefore, we conclude that the change of the number $n$ of layers within block cannot alone lead to the appearance of the maximum in the critical temperature $T_{c}$ as a function of $n$ for fixed values

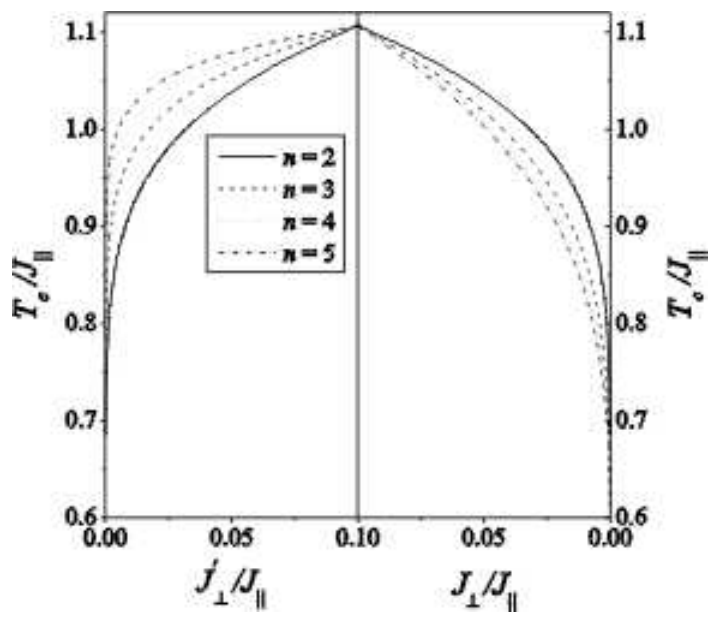

Figure 6: Critical temperature $T_{c}(n)$ as a function of $c$-axis phase stiffnesses $J_{\perp}$ and $J_{\perp}^{\prime}$ for various values of $n$ as indicated in the inset.

of the microscopic phase stiffnesses. It is necessary to introduce another factor, which acts competitively to the effective increasing of inter-layer coupling (for $J_{\perp}>J_{\perp}^{\prime}$ ) triggered by increase of $n$. As in homologous series of superconducting cuprates in-plane lattice parameters and distances between $\mathrm{Cu}-\mathrm{O}$ planes are constant among different members of the family (see, Ref. 18), one can expect that the change of $n$ will mainly affect $J_{\|}$ coupling parameter. Without assuming any specific mechanism or introducing a competing (hidden) order, one can calculate values of $J_{\|}$for given $J_{\perp}$ and $J_{\perp}^{\prime}$ leading to dependence of critical temperature on $n$, which is in agreement with experimental findings. The result is presented in Fig. 7 and in Table It Is visible that for $n=2,3$ depicted dependence changes from rising to falling. This coincides with the fact that for $n=3$ layers lose their equivalence i.e. they split for two groups: outer, lying on block surfaces and inner. Because, in real materials, within block of $\mathrm{Cu}-\mathrm{O}$ planes $c$-axis redistribution of charge is possible, one can expect that the nonequivalence of the planes within a block may lead to renormalization of the in-plane phase stiffnesses of the respective layers.

\section{CHARGE REDISTRIBUTION}

In this section, using the result of the previous paragraphs, we present a phenomenological theory for the downturn of the $T_{c}(n)$ with increasing number of layers in multilayer materials and compare it against the observed systematics, providing support to the presented theory. The idea is simple: it is well known that a superconductor is characterized by a global phase stiffness, which is proportional to the condensate density. In high$T_{c}$ cuprates the global phase stiffness is strongly doping dependent and this dependence has a universal character 


\begin{tabular}{|c|c|c|}
\hline$n$ & $T_{c}[\mathrm{~K}]$ & $J_{\|}[\mathrm{meV}]$ \\
\hline \hline 1 & 97 & 19.1 \\
\hline 2 & 126 & 19.1 \\
\hline 3 & 135 & 18.3 \\
\hline 4 & 125 & 15.8 \\
\hline 5 & 110 & 13.1 \\
\hline 6 & 97 & 11.0 \\
\hline 7 & 88 & 9.64 \\
\hline
\end{tabular}

Table II: Superconducting critical temperature $T_{c}(n)$ of $\mathrm{HgBa}_{2} \mathrm{Ca}_{n-1} \mathrm{Cu}_{n} \mathrm{O}_{2 n+2+\delta}$ compounds taken from Ref. 17 and the corresponding values of the in-plane stiffnesses $J_{\|}$ calculated from Eq. (56) for $J_{\perp}=0.261 \mathrm{meV}$ and $J_{\perp}^{\prime}=$ $0.000361 \mathrm{meV}$.

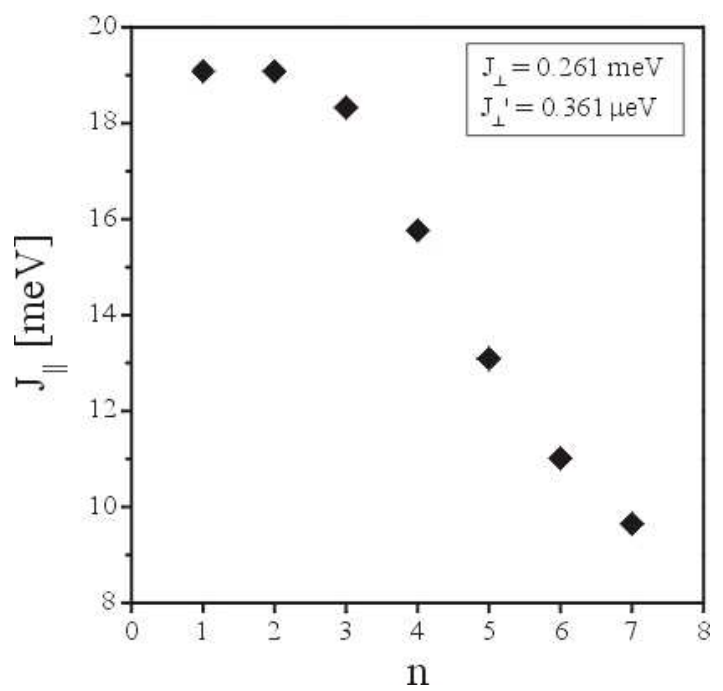

Figure 7: In-plane phase stiffness $J_{\|}$, from Table II as a function of number of layers $n$.

as evidenced experimentally by the dome-shaped dependence of the critical temperature as a function of doping. Phrased in the language of the presented model with phase variables and microscopic phase stiffnesses, it means that the parameters of this model have to be changed substantially with doping, since the global phase stiffness (i.e. the condensate) builds on microscopic phase stiffnesses and phase variables. The dependence of the phase stiffnesses on doping $\delta$ obviously cannot be found in the presented model, since they are treated as effective values to be determined, in principle, from the (yet unavailable) microscopic theory. However, assuming that all of them share the same doping dependence:

$$
\begin{aligned}
J_{\|} & =g(\delta) \tilde{J}_{\|} \\
J_{\perp} & =g(\delta) \tilde{J}_{\perp} \\
J_{\perp}^{\prime} & =g(\delta) \tilde{J}_{\perp}^{\prime},
\end{aligned}
$$

one can rewrite the saddle point equation in Eq. (56) in the form:

$$
T_{c}(\delta)=g(\delta) h\left(\tilde{J}_{\|}, \tilde{J}_{\perp}, \tilde{J}_{\perp}^{\prime}\right)
$$

where $h$ is a function that is independent on doping $\delta$. For a given microscopic phase stiffnesses it follows from Eq. (68) that the function $g(\delta)$ determines the overall doping dependence of $T_{c}(\delta)$. However, the parabolic shape of $T_{c}(\delta)$ is well-known (see, Ref. 31) and the superconducting "dome" in the $T-\delta$ phase diagram extends from $\delta \approx 0.05$ to $\delta \approx 0.25$ with the optimal doping $\delta_{\text {opt }} \approx 0.15$. This allows us to extract the doping dependence of $g(\delta)$ as a quadratic function of $\delta$ in the form:

$$
g(\delta)=\left\{\begin{array}{l}
1-\frac{1}{\alpha}\left(\delta-\delta_{\text {opt }}\right)^{2} \text { for } 0.05<\delta<0.025 \\
0 \text { for } \delta<0.05 \text { or } \delta>0.25
\end{array}\right.
$$

where $\alpha=0.01$. According to results of the previous section, in the case of strong anisotropy $\left(J_{\|} \gg J_{\perp}, J_{\perp}^{\prime}\right)$, the in-plane phase stiffness $J_{\|}$has the major influence on the critical temperature $T_{c}$, which is weakly dependent on inter-planar phase stiffnesses (see, Fig. 2). Therefore, we limit further discussion to the case, where the interplanar phase stiffnesses as functions of doping $J_{\perp} \approx \tilde{J}_{\perp}$ and $J_{\perp}^{\prime} \approx \tilde{J}_{\perp}^{\prime}$ are approximately constant. The value of $\tilde{J}_{\|}$can be determined using the following procedure. Obviously, single- and double-layer systems cannot exhibit charge redistribution: all the planes are equivalent and therefore, the layers can be optimally doped $g\left(\delta_{\text {opt }}\right)=1$. Using the data for the critical temperature $T_{c}(n)$ for optimally-doped mercuro-cuprates 17 for $n=1,2$, we can determine $\tilde{J}_{\|}$and the ratio $\tilde{J}_{\perp}^{\prime} / \tilde{J}_{\perp}$. Unfortunately, the estimates of the values of couplings along $c$-axis $\tilde{J}_{\perp}$ and $\tilde{J}_{\perp}^{\prime}$ are difficult to make. However, using the relation between zero-temperature $c$-axis and $a b$ penetration depths and bare phase stiffnesses ${ }^{32}$

$$
\begin{gathered}
\frac{1}{\lambda_{\|}^{2}(T=0)} \sim \tilde{J}_{\|} \\
\frac{1}{\lambda_{\perp}^{2}(T=0)} \sim \tilde{J}_{\perp}^{\prime}
\end{gathered}
$$

we can evaluate $\tilde{J}_{\perp}^{\prime} / \tilde{J}_{\|}$to determine the value of the

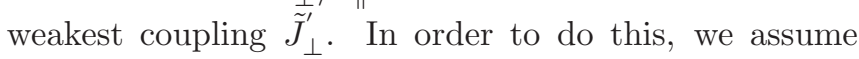
the largest anisotropy ratio

$$
\lambda_{\perp} / \lambda_{\|} \sim 230
$$

found in cuprates ${ }^{33}$ In this way all the phase stiffnesses can be fixed; we found that $\tilde{J}_{\|}=19.1 \mathrm{meV}$ (see, Table【), while $\tilde{J}_{\perp}=0.26 \mathrm{meV}$ and $\tilde{J}_{\perp}^{\prime}=0.00036 \mathrm{meV}$. Although, the estimates of $c$-axis phase stiffnesses in general can substantially vary their influence on the critical temperature is rather weak, as shown in Section II.

In the multi-layer systems $(n \geq 3)$ the planes are not equivalent any more and charge redistribution within 
blocks is possible 34.35 Therefore, in-plane phase stiffness of every layer $\ell$ becomes $J_{\|}\left(\delta_{\ell}\right)$. Since our model does not contain independent values of $J_{\|}$for different planes (which would complicate theoretical considerations considerably - given the lack of the translational symmetry along $c$-axis), we modify Eq. (67) and use the average over $n$ layers in the $n$-layer block:

$$
J_{\|} \rightarrow J_{\|}^{a v}(\delta)=\frac{\tilde{J}_{\|}}{n} \sum_{i=1}^{n} g\left(\delta_{i}\right) .
$$

However, nuclear magnetic resonance (NMR) measurements show that charge distribution among inner planes is almost constant ${ }^{34}$ Therefore, we characterize charge distribution by two parameters: we assume that the doping of inner and outer planes is given by $\delta_{i p}$ and $\delta_{o p}$, respectively, see Fig. 8 Introducing a ratio of dopings for outer and inner planes

$$
R=\delta_{o p} / \delta_{i p}>1,
$$

and noting that in every block there are 2 outer and $n-2$ inner layers, we can write Eq. (72) as:

$$
J_{\|}^{a v}\left(\delta_{i p}, R, n\right)=\frac{\tilde{J}_{\|}}{n}\left[2 g\left(R \delta_{i p}\right)+(n-2) g\left(\delta_{i p}\right)\right] .
$$

By observing that the maximum of the critical temperature $T_{c}(n)$ corresponds to the maximum of $J_{\|}^{a v}\left(\delta_{i p}, R, n\right)$, we can find the optimal value of $\delta_{i p}=\delta_{o p t}^{*}$ using the condition

$$
\left.\frac{d J_{\|}^{a v}\left(\delta_{i p}, R, n\right)}{d \delta_{i p}}\right|_{\delta_{i p}=\delta_{o p t}^{*}}=0 .
$$

Explicitly, using Eq. (74) we obtain for $\delta_{o p t}^{*}$ :

$$
\delta_{o p t}^{*}=\delta_{o p t}^{2} \frac{n-2+2 R}{n-2+2 R^{2}},
$$

which allows to find the value of the effective in-plane phase stiffness

$$
J_{\|} \equiv J_{\|}^{a v}(n, R)
$$

as a function of number of layers $n$ and charge imbalance ratio $R$ :

$$
J_{\|}^{a v}(n, R)=\tilde{J}_{\|}\left[1-\frac{\delta_{o p t}^{2}(n-2)(R-1)^{2}}{\alpha n\left(n-2+2 R^{2}\right)}\right] .
$$

Using Eq. (78) we can determine the charge imbalance parameters $R$ as a function of number of layers $n$ for a given set of experimental data for the critical temperature $T_{c}(n)$. For this purpose we use the dependence of the superconducting critical temperature on number of layers reported by Kuzemskaya, et al., Ref. 17. The procedure reads as follows:

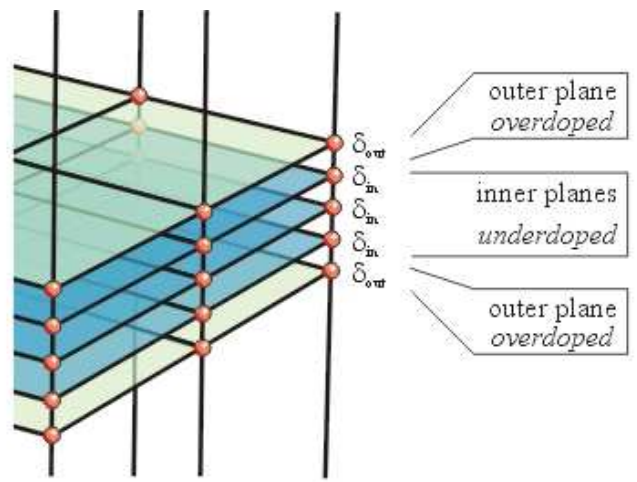

Figure 8: (Color online) Schematic representation of charge redistribution leading to overdoping of outer and underdoping of inner layers, respectively.

(i) For $n=1$ and $n=2$ by definition $R=1$ and consequently $J_{\|}^{a v}(n, R) \equiv \tilde{J}_{\|}$. Using the value of $T_{c}(1)$ from experiment, we can determine the in-plane $\tilde{J}_{\|}$and $\tilde{J}_{\perp}^{\prime} \equiv \tilde{J}_{\perp}$ (for $n=1$ ) phase stiffnesses using Eq. (56) for the critical temperature $T_{c}(n=1)$ and the supplementary equations (70) and (71).

(ii) For $n=2$ we determine in-block $\tilde{J}_{\perp}$ phase stiffness using Eq. (56) for $T_{c}(n=2)$, while keeping $\tilde{J}_{\|}$and $\tilde{J}_{\perp}^{\prime}$ unchanged. In this way all the energy setting parameters $\tilde{J}_{\|}, \tilde{J}_{\perp}$ and $\tilde{J}_{\perp}^{\prime}$ are fixed.

(iii) For $n>2$, with the computed parameters $\tilde{J}_{\|}, \tilde{J}_{\perp}$ and $\tilde{J}_{\perp}^{\prime}$, by making use of Eq. (56) for theoretical $T_{c}(n)$, we calculate the values of $R$, using $T_{c}(n)$ as an input from experiment.

The obtained values of the ratio $R$ are presented in Fig. 9] while resulting dopings of inner and outer planes are shown in Fig. 10. From these results we can infer that for $n=1,2,3$ the critical temperature is rising due to the increased inter-layer (intra-block) phase stiffness $J_{\perp}>J_{\perp}^{\prime}$, while the charge redistribution for $n=3$ is rather small. For values of $n>3$ the critical temperature is very sensitive to charge redistribution and we have a decrease from $T_{c}=135 \mathrm{~K}$ for $n=3(R=1.35)$ to $T_{c}=125 \mathrm{~K}$ for $n=4(R=1.82)$. The calculated values of $R$ are in good agreement with experimental results based on NMR measurement of $\mathrm{HgBa}_{2} \mathrm{Ca}_{4} \mathrm{Cu}_{5} \mathrm{O}_{y}$ compound by Kotegawa, Ref. 34. Interestingly, the obtained parameters $R$ for $n=3,4$ are much smaller than those found in Ref. 35 ( $R=4.5$ and $R=9$, respectively) calculated using simplified considerations based on competition between electrostatic and band energy estimates. However, in the real materials charge depletion in the inner layers may be high enough to destroy the superconductivity in these layers, i.e. doping of one type of planes may be well outside superconducting region. This scenario have also been confirmed experimentally by NMR studies of fivelayer compound $\mathrm{HgBa}_{2} \mathrm{Ca}_{4} \mathrm{Cu}_{5} \mathrm{O}_{y}, \underline{36}$ Only outer $\mathrm{Cu}-\mathrm{O}$ planes are superconducting with $T_{c}=108 K$, while inner planes exhibit a static antiferromagnetic ordering with 


\begin{tabular}{|c|c|c|c|c|}
\hline$n$ & $R$ (this work) & $R$ (Ref. 34) & $R$ (Ref. 35) & $R$ (Ref. 36) \\
\hline \hline 1 & 1 & 1 & 1 & n.a. \\
\hline 2 & 1 & 1 & 1 & n.a. \\
\hline 3 & 1.35 & 1.14 & $4.5^{a}$ & n.a. \\
\hline 4 & 1.82 & 1.49 & $9^{b}$ & n.a. \\
\hline 5 & 2.23 & 1.64 & n.a. & $3.8^{c}$ \\
\hline 6 & 2.56 & n.a. & n.a. & n.a. \\
\hline 7 & 2.78 & n.a. & n.a. & n.a. \\
\hline
\end{tabular}

Table III: Calculated values of the ratio $R$ (this work, second column) as a function of number of layers $n$ using the data for $T_{c}(n)$ from Kuzemskaya, et al. Ref. 17, compared with the results from other works: $R$ based on calculated values of charge concentration of outer $\left(0.45^{a, b}\right)$ and inner layers $\left(0.1^{a}, 0.05^{b}\right)$ using a theory of competition between electrostatic and band energy estimates (Di Stasio, et al., Ref. 35); nuclear magnetic resonance measurement of $\mathrm{HgBa}_{2} \mathrm{Ca}_{4} \mathrm{Cu}_{5} \mathrm{O}_{y}$ compound ${ }^{c}$ with the values of charge concentration of outer (0.212) and inner layers (0.057); n.a. - non-available.

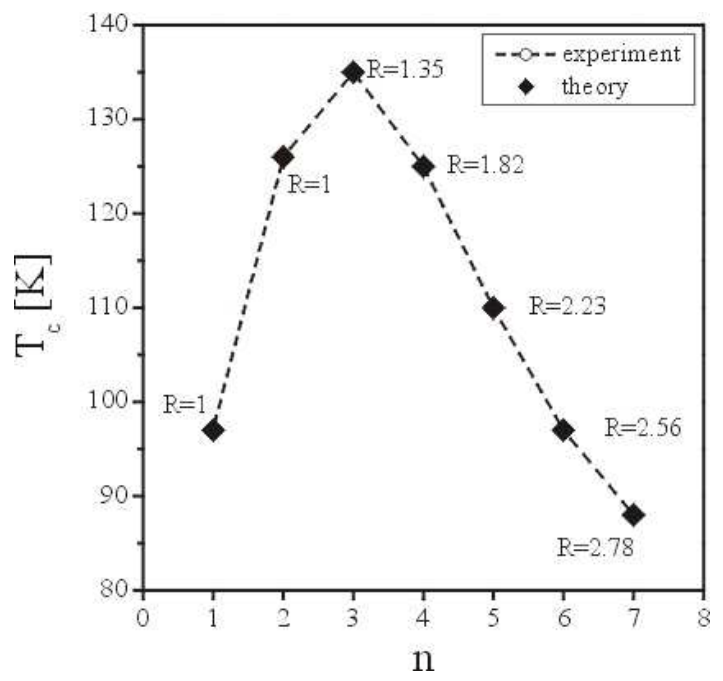

Figure 9: Critical temperature $T_{c}$ vs. number of layers $n$ that fits the experimental data from Ref. 17 and the calculated values of parameter $R$ describing the ratio of doping of outer to inner planes. Dashed line is only guide for the eye.

$T_{N}=60 \mathrm{~K}$. This suggest possible extension of our model to include independent in-plane phase stiffnesses of the inner and outer layers.

\section{SUMMARY AND CONCLUSIONS}

We have considered a model of $n$-layer hightemperature cuprates of homologous series like $\mathrm{HgBa}_{2} \mathrm{Ca}_{n-1} \mathrm{Cu}_{n} \mathrm{O}_{2+2 n+\delta}$ to study the dependence of the critical temperature $T_{c}(n)$ on the number $n$ of $\mathrm{Cu}-\mathrm{O}$ planes in the elementary cell. We focus on the "phase only" description of the high-temperature superconducting system motivated by the experimental

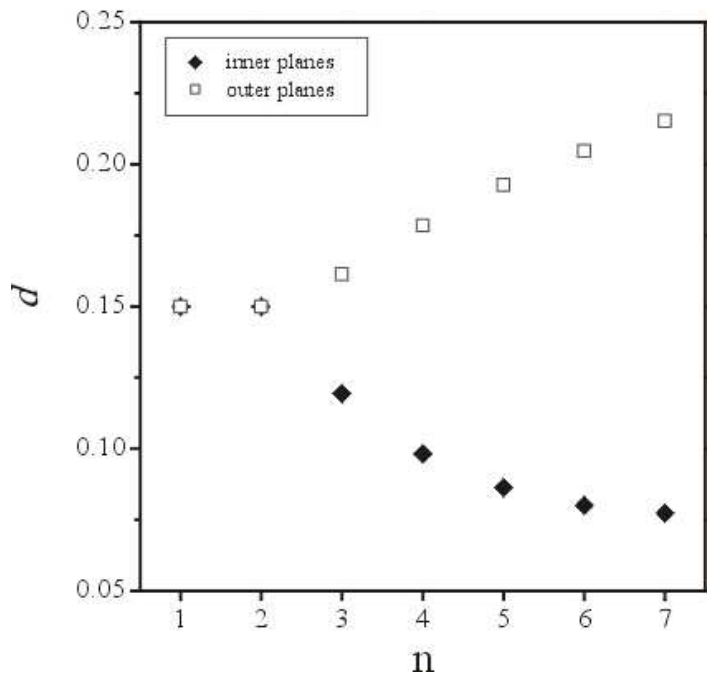

Figure 10: Doping of inner $\delta_{i p}$ and outer $\delta_{o p}$ planes vs. number of layers $n$ that corresponds to the data from Fig. 9

evidence that the ordering of the phase degrees of freedom is responsible for the emergence of the superconducting state with long-range order. To this end, we have proposed a three-dimensional semi microscopic $\mathrm{XY}$ model with two-component vectors that involve phase variables and adjustable parameters representing microscopic phase stiffnesses. The model fully implements complicated stacked plane structure along $c$-axis to capture the layered composition of homologous series. This allows us to go well beyond widely used phenomenological Lawrence-Doniach model for layered superconductors. Implementing spherical closure relation we have solved the phase XY model exactly with the help of transfer matrix method and calculated $T_{c}(n)$ for chosen system parameters and arbitrary block size $n$. In this way we were able to elucidate the role of the $c$-axis anisotropy and its influence on the critical temperature. Furthermore, by making a physically justified assumption regarding the doping dependence of the microscopic phase stiffnesses we were able to calculate the bell shaped curve of $T_{c}(n)$ as a function of block size $n$ with a maximum at $n=3$ by effectively accommodating inhomogeneous charge distribution among planes. The success of our approach suggests to us that at least for the superconducting phase transition properties studied, one does not need to invoke any competing "hidden" order parameter that appear in other approaches ${ }^{22} \mathrm{In}$ the present work, the vanishing of the superconductivity with underdoping can be understood by the reduction of the in-plane phase stiffnesses and can be linked to the manifestation of Mott physics (no double occupancy due to the large Coulomb on-site repulsion) that leads to the loss of long-range phase coherence while moving toward half-filled limit $(\delta=0)$. For doping $\delta=0$, the fixed electron number implies large fluctuations in the conjugate phase variable, which naturally translates into the reduction of the microscopic in-plane phase stiffnesses 
and destruction of the superconducting long-range order in this limit. In the opposite region of large $\delta$, the onset of a pair-breaking effect (at the pseudogap temperature $\left.T^{*}\right)$ can deplete the microscopic phase stiffnesses, thus reducing the critical temperature. Therefore, it would be interesting to relate the phase stiffnesses parameters used in the present paper with the microscopic material characteristics of the electronic system (like hopping parameters, antiferromagnetic exchange and Coulomb energy) to establish a link between our semi-microscopic approach and physics of strongly correlated systems. The charge redistribution taking place in multi-layered cuprates also points out the profound impact of charging effects and unusual role of the internal electric fields in cuprate superconductors. $\frac{37}{6}$

\section{Acknowledgments}

T. A. Zaleski would like to thank The Foundation for Polish Science for support.

\section{Appendix A: MATRIX DIAGONALIZATION}

The transfer matrix method requires raising transfer matrix to an arbitrary power. If matrix $\mathbf{T}$ of the order $m$ has $m$ distinct eigenvalues $\lambda_{1}, \ldots, \lambda_{m}$ it can be diagonalized:

$$
\mathbf{T}=\mathcal{U D} \mathcal{U}^{-1}
$$

where $\mathcal{U}$ is a matrix built of eigenvectors $\hat{\varepsilon}_{1}, \ldots, \hat{\varepsilon}_{m}$ of $\mathbf{T}$ :

$$
\mathcal{U}=\llbracket \hat{\varepsilon}_{1} \ldots \hat{\varepsilon}_{m}
$$

while

$$
\mathcal{D}=\left[\begin{array}{ccc}
\lambda_{1} & 0 & 0 \\
0 & \ddots & 0 \\
0 & 0 & \lambda_{m}
\end{array}\right]
$$

is diagonal matrix. In result any integer power $p$ of $\mathbf{T}$ can be simply found:

$$
\mathbf{T}^{p}=\underbrace{\left(\mathcal{U} \mathcal{D} \mathcal{U}^{-1}\right) \ldots\left(\mathcal{U D} \mathcal{U}^{-1}\right)}_{p}=\mathcal{U} \mathcal{D}^{p} \mathcal{U}^{-1}
$$

since $\mathcal{U}^{-1} \mathcal{U}=1$. Finally:

$$
\mathbf{T}^{p}=\mathcal{U}\left[\begin{array}{ccc}
\lambda_{1}^{p} & 0 & 0 \\
0 & \ddots & 0 \\
0 & 0 & \lambda_{m}^{p}
\end{array}\right] \mathcal{U}^{-1}
$$

This procedure is utilised in Sec. III. The columns of the matrix $\mathcal{U}_{1}$ in Eq. 42 contain eigenvectors of matrix $\mathbf{T}_{1}$ :

$$
\mathcal{U}_{1}=\left[\begin{array}{cc}
\frac{4\left|B_{n}\right|+\left(\beta J_{\perp}^{\prime}\right)^{2}\left|B_{n-2}\right|-\sqrt{\Delta}}{8\left|B_{n-1}\right|} & \frac{4\left|B_{n}\right|+\left(\beta J_{\perp}^{\prime}\right)^{2}\left|B_{n-2}\right|+\sqrt{\Delta}}{8\left|B_{n-1}\right|} \\
1 & 1
\end{array}\right]
$$

where:

$$
\Delta=\left[16\left|B_{n}\right|+\left(\beta J_{\perp}^{\prime}\right)^{2}\left|B_{n-2}\right|\right]^{2}-\left(4 \beta J_{\perp}^{\prime}\left|B_{n-1}\right|\right)^{2}
$$

The inverse of the matrix $\mathcal{U}_{1}$ is given by:

$$
\mathcal{U}_{1}^{-1}=\left[\begin{array}{cc}
-\frac{4\left|B_{n-1}\right|}{\sqrt{\Delta}} & \frac{4\left|B_{n}\right|+\left(\beta J_{\perp}^{\prime}\right)^{2}\left|B_{n-2}\right|+\sqrt{\Delta}}{2\left|B_{n-1}\right|} \\
\frac{4\left|B_{n-1}\right|}{\sqrt{\Delta}} & -\frac{4\left|B_{n}\right|+\left(\beta J_{\perp}^{\prime}\right)^{2}\left|B_{n-2}\right|-\sqrt{\Delta}}{2\left|B_{n-1}\right|}
\end{array}\right] .
$$

Similarly, the matrix $\mathcal{U}_{2}$ in Eq. $\left[50\right.$ is built of eigenvectors of $\mathbf{T}_{2}$ :

$$
\mathcal{U}_{1}=\left[\begin{array}{cc}
\frac{1}{2}\left(\zeta-\frac{\beta J_{\|}}{2}\right)-\frac{1}{2} \sqrt{\left[\left(\zeta-\frac{\beta J_{\|}}{2}\right)^{2}-\left(\beta J_{\perp}\right)^{2}\right]} & \left.\frac{1}{2}\left(\zeta-\frac{\beta J_{\|}}{2}\right)+\frac{1}{2} \sqrt{\left[\left(\zeta-\frac{\beta J_{\|}}{2}\right)^{2}-\left(\beta J_{\perp}\right)^{2}\right]}\right] \\
1
\end{array}\right.
$$


1 J.G. Bednorz and K.A. Müller, Z. Phys. B 64, 189 (1986)

${ }^{2}$ P. W. Anderson, The Theory of Superconductivity in the High $T_{c}$ Cuprates (Princeton University Press, Princeton, NJ, 1995).

3 A. J. Leggett, Science 274, 587 (1996).

4 X. D. Xiang, A. Zettl, W. A. Vareka, J. L. Corkill, T. W. Barbee III, M. L. Cohen, Phys. Rev. B 43, 11496 (1991).

5 W. E. Lawrence, S. Doniach in: Proceedings of the 12th International Conference on Low Temperature Physics, p. 361, Ed. E. Kanda, Academic Press of Japan, Kyoto, 1971.

6 M. Machida, T. Koyama, M. Tachiki, Phys. Rev. Lett. 83, 4618 (1999).

7 L. N. Bulaevskii, I. D. Vagner, Phys. Rev. B 43, 8694 (1991)

8 V. P. Mineev, Phys. Rev. B 65, 012508 (2002).

9 S. V. Kuplevakhsky, Phys. Rev. B 60, 7496 (1999); Phys. Rev. B 63, 054508 (2001); V. M. Krasnov, Phys. Rev. B 65, 096503 (2002).

10 V. J. Emery, S. A. Kivelson, Nature 374, 434 (1995).

11 J. M. Wheatley, T. C. Hsu, P. W. Anderson, Nature 333, 121(1988); S. Chakravarty, A. Sudbo, P. W. Anderson, S. Strong, Science 261, 337 (1993); P. W. Anderson, Science 268, 1154 (1995).

12 K.A. Moler, J.R. Kirtley, D.G. Hinks, T.W. Li, and M. Xu, Science 279, 1193 (1998).

13 A.A. Tsvetkov, D. van der Marel, K.A. Moler, J.R. Kirtley, J.L. de Boer, A. Meetsma, Z.F. Ren, N. Koleshnikov, D. Dulic, A. Damascelli, M. Grninger, J. Schtzmann, J.W. van der Eb, H.S. Somal, and J.H. Wang, Nature (London) 395, 360 (1998).

14 S. Chakravarty, H. -Y. Kee, E. Abrahams, Phys. Rev. Lett. 82, 2366 (1999); T. Schneider, Physica B 326, 289 (2003).

15 J.-H. Choy, S.-J. Kwon, and G.-S. Park, Science 280, 1589 (1998).

16 X.-D. Xiang, S. McKernan, W.A. Vareka, A. Zettl, J.L. Corkill, T.W. Barbee III, and M.L. Cohen, Nature (London) 348, 145 (1990).

17 I. G. Kuzemskaya, A. L. Kuzemsky, and A. A. Cheglokov, J. Low Temp. Phys. 118, 147 (2000).

18 B. A. Scott, E. Y. Suard, C. C. Tsuei, D. B. Mitzi, T. R. McGuire, B. -H. Chen, D. Walker, Physica C 230, 239 (1994).

19 J. M. Wheatley, T. C. Hsu, and P. W. Anderson, Phys. Rev. B 37, 5897 (1988).

20 A. J. Leggett, Phys. Rev. Lett. 83, 392 (1999); Phys. Rev. Lett. 85, 3984 (2000); S. Chakravarty, Eur. Phys. J. B 5, 337 (1998); there are other forms of the enhancement formula, which give only slightly different results and are discussed in the following review: L. Jansen and R. Block,
Physica A 289, 165 (2001).

21 E. S. Itskevich, E. V. Zhasinas, Physica C 295, 193 (1998).

22 S. Chakravarty, H.-Y. Kee, and K. Völker, Nature 428, 53 (2004).

23 S. Chakravarty, R. B. Laughlin, D. K. Morr, C. Nayak, Phys. Rev. B 63, 094503 (2001).

24 J. Corson, R. Mallozzi, J. Orenstein, J. N. Eckstein, I. Bozovic, Nature 398, 221 (1999).

25 T. H. Berlin and M. Kac, Phys. Rev. 86, 821 (1952); H. E. Stanley, ibid. 176, 718 (1968); G. S. Joyce, Phys. Rev. 146, 349 (1966); G. S. Joyce, in Phase Transitions and Critical Phenomena, edited by C. Domb and M. S. Green (Academic, New York, 1972), Vol. 2, p. 375.

26 The spherical model was originally proposed for onedimensional Ising variables. In the present paper, vectors $\mathbf{S}_{\ell}\left(\mathbf{r}_{i}\right)$ are two-dimensional, which implies that the presented model is a spherical version of a $M$-vector model with a fixed $M=2$. The spherical model earned wider attention after calculations based on high-temperature series expansion proved it was reasonable approximation of the Heisenberg three-dimensional system, see P. S. English, D. L. Hunter, C. Domb, J. Phys. A 12 (1979) 2111; H. E. Stanley, J. App. Phys. 40 (1969) 1272.

27 Handbook of Mathematical Functions, edited by $\mathrm{M}$. Abramovitz and I. Stegun Dover, New York, 1970.

28 S. Fishman, T. A. L. Ziman, Phys. Rev. B 26, 1258 (1982).

29 E. W. Carlson, S. A. Kivelson, V. J. Emery, and E. Manousakis, Phys. Rev. Lett. 83, 612 (1999).

30 K. Binder, Phys. Rev. Lett. 47, 693 (1981).

31 V. M. Krasnov, Phys. Rev. B 65, 140504 (2002) .

32 E. Roddick, D. Stroud, Phys. Rev. Lett. 74, 1430 (1995).

${ }^{33}$ It is difficult in general to determine the value $J_{\perp}^{\prime} / J_{\|}$, therefore we made an estimate, which relies on the experimental data for a high- $T_{c}$ system (BSCCO) with the largest $\lambda_{\perp} / \lambda_{\|}$anisotropy, see N.E. Hussey, J.R. Cooper, R.A. Doyle, C.T. Lin,W.Y. Liang, D.C. Sinclair, G. Balakrishnan, D.McK. Paul, A. Revcolevschi, Phys. Rev. B 53, 6752 (1996).

34 H. Kotegawa, Y. Tokunaga, K. Ishida, G.-q. Zheng, Y. Kitaoka, K. Asayama, H. Kito, A. Iyo, H. Ihara, K. Tanaka, K. Tokiwa, T. Watanabe, J. Phys. Chem. Solids 62, 171 (2001).

35 M. Di Stasio, K. A. Müller, and L. Pietronero, Phys. Rev. Lett. 64, 2827 (1990).

36 H. Kotegawa, Y. Tokunaga, Y. Araki, G.-q. Zheng, Y. Kitaoka, K. Tokiwa, K. Ito, T. Watanabe, A. Iyo, Y. Tanaka, H. Ihara, Phys. Rev. B 69, 014501 (2004).

37 J. E. Hirsch, Phys. Rev. Lett. 92, 016402 (2004). 